\title{
Prelimbic and Infralimbic Prefrontal Regulation of Active and Inhibitory Avoidance and Reward-Seeking
}

\author{
Giulia Capuzzo and ${ }^{\circledR}$ Stan B. Floresco \\ Department of Psychology and Djavad Mowafaghian Centre for Brain Health, University of British Columbia, Vancouver, B.C., Canada V6T 1Z3
}

Flexible initiation or suppression of actions to avoid aversive events is crucial for survival. The prelimbic (PL) and infralimbic (IL) regions of the medial prefrontal cortex (mPFC) have been implicated in different aspects of avoidance and reward-seeking, but their respective contribution in instigating versus suppressing actions in aversive contexts remains to be clarified. We examined mPFC involvement in different forms of avoidance in rats well trained on different cued lever-press avoidance tasks. Active/inhibitory avoidance required flexible discrimination between auditory cues signaling foot-shock could be avoided by making or withholding instrumental responses. On a simpler active avoidance task, a single cue signaled when a lever press would avoid shock. PL inactivation disrupted active but not inhibitory avoidance on the discriminative task while having no effect on single-cued avoidance. In comparison, IL inactivation broadly impaired active and inhibitory avoidance. Conversely, on a cued appetitive go/no-go task, both IL and PL inactivation impaired inhibitory but not active reward-seeking, the latter effect being diametrically opposite to that observed on the avoidance task. These findings highlight the complex manner in which different mPFC regions aid in initiating or inhibiting actions in the service of avoiding aversive outcomes or obtaining rewarding ones. IL facilitates active avoidance but suppress inappropriate actions in appetitive and aversive contexts. In contrast, contextual valence plays a critical role in how the PL is recruited in initiating or suppressing actions, which may relate to the degree of cognitive control required to flexibly negotiate response or motivational conflicts and override prepotent behaviors.

Key words: avoidance; cognitive control; prefrontal cortex; rat; response inhibition

Significance Statement

Choosing to make or withhold actions in a context-appropriate manner to avoid aversive events or obtain other goals is a critical survival skill. Different medial prefrontal cortex (mPFC) regions have been implicated in certain aspects of avoidance, but their contributions to instigating or suppressing actions remains to be clarified. Here, we show that the dorsal, prelimbic (PL) region of the medial PFC aids active avoidance in situations requiring flexible mitigation of response conflicts, but also aids in withholding responses to obtain rewards. In comparison the ventral infralimbic (IL) cortex plays a broader role in active and inhibitory avoidance as well as suppressing actions to obtain rewards. These findings provide insight into mechanisms underlying normal and maladaptive avoidance behaviors and response inhibition.

\section{Introduction}

Selecting appropriate actions to deal with environmental threats and avoid potential negative consequences is an essential class of defensive behaviors. Often, threat avoidance requires instigation of actions based on innate or learned associations between cues

Received Feb. 20, 2020; revised Apr. 23, 2020; accepted May 3, 2020.

Author contributions: G.C. and S.B.F. designed research; G.C. performed research; G.C. and S.B.F. analyzed data; G.C. and S.B.F. wrote the paper.

This work was supported by a Discovery Grant (RGPIN-2018-04295) from the Natural Sciences and Engineering Research Council of Canada (to S.B.F.). We thank Dr. Patrick Piantadosi for his assistance in implementing these studies, Jenna Stein for assistance with behavioral testing and Dr. David Moorman for his invaluable comments on this manuscript.

The authors declare no competing financial interests.

Correspondence should be addressed to Stan B. Floresco at floresco@psych.ubc.ca.

https://doi.org/10.1523/JNEUROSCI.0414-20.2020

Copyright $\odot 2020$ the authors predictive of impending danger (active avoidance), whereas in other situations, aversive consequences may be avoided by constraining actions in response to threat stimuli (passive or inhibitory avoidance).

Recently, there has been a resurrection of interest in examining the neural circuitry mediating expression of different forms of avoidance, primarily focusing on active avoidance (LeDoux et al., 2017). For example, lateral amygdala nuclei facilitate acquisition and expression of a shuttling avoidance response prompted by an auditory warning cue, and do so via interactions with the shell (but not core) subregion of the nucleus accumbens (NAc; Ramirez et al., 2015). In comparison, inactivation of the lateral amygdala or NAc core disrupts signaled "platform-mediated" avoidance, requiring rats to disengage from reward-seeking and move to a safe location (Bravo-Rivera et al., 2014). More recently, work by our group has shown that NAc core and shell play 
cooperative but distinct roles in mediating performance of an active/inhibitory avoidance task, in which different cues informed rats that a lever press should either be emitted or withheld to avoid shock. Under these conditions, the NAc core is involved selectively in expression of active avoidance, whereas the shell modulates both active and inhibitory avoidance (Piantadosi et al., 2018).

The amygdala and NAc are interconnected with the prelimbic (PL) and infralimbic (IL) regions of the medial prefrontal cortex (mPFC; Hurley et al., 1991; Brog et al., 1993; Groenewegen et al., 1999; Vertes, 2004), and these regions have also been implicated in avoidance, although studies have yielded somewhat conflicting findings. For example, earlier studies reported that large lesions of the mPFC either impaired, had no effect, or slightly improved acquisition of avoidance responses, depending on experimental conditions (Brennan and Wisniewski, 1982; Holson, 1986; Brito and Brito, 1990; Joel et al., 1997). More recent studies revealed that IL inactivation perturbed expression of shuttle avoidance and caused a corresponding disinhibition of freezing, whereas PL lesions had no effect on either measure (Moscarello and LeDoux, 2013). In contrast, PL inactivation disrupted platform-mediated avoidance without affecting freezing, whereas IL inactivation increased freezing without affecting avoidance (Bravo-Rivera et al., 2014).

These results suggest that specific prefrontal-subcortical circuits play cooperative but distinct roles in different forms of avoidance and may be particularly important in situations involving response conflict that require flexible action selection. This is in keeping with the proposed involvement of the mPFC in cognitive control, mediating conflicting approach versus avoidance behaviors and under some conditions, suppressing behaviors that may lead to negative consequences (Chudasama and Robbins, 2003; Bari and Robbins, 2013; Burgos-Robles et al., 2017; Schwartz et al., 2017; Verharen et al., 2019). However, how these regions may differentially contribute to action selection in situations that require shifts between making and inhibiting actions to avoid negative events remains to be clarified. To address this, we examined how the PL and IL enable expression of opposing forms of signaled avoidance using an aversive go/ no-go task, wherein rats were well trained to discriminate between different auditory cues and shift between instigation and suppression of actions. This active/inhibitory avoidance task was patterned after those used in studies with humans, revealing activation of corticolimbic circuitry in situations requiring response initiation or inhibition to avoid negative consequences (Schlund et al., 2011, 2016; Levita et al., 2012). Moreover, performance of this task in rats is dependent on neural activity within different subregions of the NAc, a main target of mPFC outputs (Piantadosi et al., 2018). We then parsed out the discriminative component of this signaled avoidance task, examining how the PL and IL mediate behavior in a simpler active avoidance situation, when only a single cue signaled a lever press could avoid shock. The results of these experiments prompted additional investigation into the involvement of these two regions in initiating or inhibiting instrumental responses in the context of reward-seeking, to ascertain how the valence of the outcome (avoiding shock vs obtaining reward) may differentially influence how these regions contribute to aiding or inhibiting instrumental action.

\section{Materials and Methods}

\section{Animals}

All experimental procedures were approved by the Animal Care Committee at the University of British Columbia and conducted in compliance with guidelines provided by the Canadian Council on Animal Care. All reasonable efforts were made to minimize the number of animals used and their suffering. In these studies, male Long-Evans rats were used. There have been reports that male versus female rats display differences in the acquisition and extinction of active and passive avoidance (Heinsbroek et al., 1988; Beck et al., 2011). In light of the complexity of the behavioral procedures and the multiple brain regions targeted in this initial exploration of mPFC mediation of active and inhibitory avoidance, we opted to simplify our experimental design and not include sex differences as an additional variable in our analyses.

Rat arrived at the vivarium weighing 250-275 g (Charles River) and were housed in groups of four per cage for about a week with ad libitum access to food and water to allow acclimation and were handled daily. They were then split into pairs of similar weight and food restricted to $\sim 90 \%$ of their free feeding weight starting $2-3 \mathrm{~d}$ before behavioral training and allowed to gain weight throughout the course of the experiment on a delayed-growth curve ( $\sim-10 \mathrm{~g} /$ week). For the avoidance experiments, food restriction was instituted to encourage exploration of the conditioning chambers. Food consisted of standard laboratory rat chow delivered in the home cage. Colony temperature $\left(21^{\circ} \mathrm{C}\right)$ and light cycle $(12 / 12 \mathrm{~h}$ light/dark) were kept constant. Training and handling occurred during the light hours.

\section{Apparatus}

Behavioral testing was conducted in operant chambers $(30.5 \times 24 \times$ $21 \mathrm{~cm}$, Med-Associates). Each chamber was equipped with a fan to provide ventilation and attenuation of external noise. As viewed from the opening door, two levers were situated on the right wall of each chamber and were separated by a food receptacle from which sucrose pellet reinforcement was delivered (45-mg pellet, BioServ). For all experiments, only the left lever was extended into the chamber. All chambers had three 100-mA cue lights: one over each retractable lever and a central one over the food receptacle. An additional 100-mA house light was situated on the wall opposite the food receptacle. The floor of the chambers consisted in a grid of 19 parallel stainless-steel rods spaced $1.5 \mathrm{~cm}$ apart, wired to a shock generator and solid-state grid scrambler for the delivery of foot-shock. Four infrared photobeams located just above the floors on the front and back walls, and photobeam breaks were used as an index of locomotor activity. All data were recorded on a computer connected to the chambers.

\section{Surgery}

Animals were single housed and allowed ad libitum food without training for $3 \mathrm{~d}$ before surgery. They were injected a subanesthetic dose of ketamine $(50 \mathrm{mg} / \mathrm{kg})$ and xylazine $(5 \mathrm{mg} / \mathrm{kg})$ intraperitoneally and maintained on inhalant isoflurane (1-3\%) throughout surgery. Rats were secured with earbars (flat skull) in a stereotaxic frame and analgesia was administered subcutaneously (Anafen, $10 \mathrm{mg} / \mathrm{kg}$ ). They were then implanted with a set of 23-gauge bilateral stainless-steel guide cannula. The guide cannulae were beveled at the tip to minimize damage when implanted, which in turn would be expected to curtail backflow of infusate to more dorsal regions. Cannulae were lowered vertically into the PL or IL according to the following stereotaxic coordinates: PL, from bregma: anterior/posterior $+3.2 \mathrm{~mm}$; medial/lateral: $\pm 0.7 \mathrm{~mm}$; from dura: dorsal/ventral: $-2.8 \mathrm{~mm}$ (from dura) and IL, from bregma: anterior/posterior: $+2.8 \mathrm{~mm}$; medial/lateral: $\pm 0.7 \mathrm{~mm}$; from dura: dorsal/ ventral: $-4.1 \mathrm{~mm}$ (from dura).

Cannulae were held in place by dental cement adhering to four stainless-steel skull screws at the edges of the incision. Stainless-steel 30-gauge obturators flush with the end of the guides were inserted and remained in place until infusions. The animals were given daily analgesia (Anafen, $10 \mathrm{mg} / \mathrm{kg}$, s.c.) for $2 \mathrm{~d}$ following surgery. Rats were given $5-7 \mathrm{~d}$ to recover from surgery before resuming or beginning behavioral training.

\section{Behavioral training}

Active/inhibitory avoidance training was similar to that reported by Piantadosi et al. (2018) and based on a paradigm used in humans, as described by Levita et al. (2012). Animals trained on the full version of the task (active/inhibitory avoidance) and on the single-cue active 
avoidance variation began training shortly after arrival at the colony, whereas those allocated to the active/inhibitory reward-seeking experiment received surgery before training. Rats received daily training sessions 5-7 d/week between 9 A.M. and 1 P.M. and were fed after each session.

\section{Familiarization with lever pressing}

On the first day of training, rats were placed in the chambers for $1 \mathrm{~h}$, with the house light illuminated, but no levers or auditory stimuli were presented. Rats were permitted familiarize themselves with the chamber to reduce neophobia associated with this novel environment.

Rats then received non-reinforced lever press training (Fernando et al., 2015). At the start of these 1-h sessions, the house light was illuminated and the lever on the left of the food port (the one further from the entry door) was extended. If a rat pressed the lever, this caused it to retract for $1 \mathrm{~s}$, after which it was reinserted. There was no limit to the number of presses animals could make during a session, and this procedure allowed rats to learn about the nature of the manipulandum. Rats received daily sessions until they reached criteria of $>40$ presses in a session or after $4 \mathrm{~d}$ of training. If a rat did not reach criteria after two sessions, sucrose powder was placed on the lever to entice approach and contact with the lever, although lever pressing was never explicitly reinforced with sucrose delivery. Rats performed a mean of $87 \pm 11.36$ lever presses during their final lever-retraction training session (range 40-673 presses). Six rats whose data were included in the final analysis of the active avoidance cohort did not reach criteria after $4 \mathrm{~d}$ of training but were still advanced to next phase of training (range 0-30 presses).

\section{Single-cue active avoidance training}

Rats then started training on the first phase of avoidance instrumental learning. Daily sessions consisted of 20 active avoidance trials with a variable $105 \mathrm{~s}( \pm 30 \mathrm{~s})$ intertrial interval (ITI). For all tasks used in this study, the lever was retracted during the ITI. At the beginning of each trial, extension of the left lever coincided with the presentation of an auditory warning cue. For these experiments, the cue initially associated with active avoidance was white noise $(80 \mathrm{~dB})$, as previous observations in our laboratory revealed that rats acquire active lever-press avoidance more rapidly when using this versus a high pitch pure tone $(9 \mathrm{kHz}$, $80 \mathrm{~dB}$; Piantadosi et al., 2018; and our unpublished observations). If a rat pressed the lever within $15 \mathrm{~s}$ of its insertion, this terminated the sound, retracted the lever and the trial was recorded as a "successful active avoidance." In addition, a successful active avoidance lever press turned off the house light and illuminated a central stimulus light on the same wall as the lever for $30 \mathrm{~s}$, which served as a safety signal to reinforce the operant response. Presentation of a safety signal reinforces avoidance learning by explicitly signaling successful avoidance, and thus, safety (Berger and Brush, 1975; Dinsmoor, 2001; Fernando et al., 2014, 2015). These 30-s periods were added onto the ITI, so that, depending on the number of successful avoidance responses, sessions could last between 40-50 min.

Failure to press lever within the first $15 \mathrm{~s}$ led to a subsequent 10 -s escape period. Here, the lever remained inserted, but the auditory cue was terminated and the first of up to 3, 0.5-s foot-shocks were delivered. If no lever press was made after the first shock, two additional footshocks were delivered after 5 and $10 \mathrm{~s}$ (i.e., 25 and $30 \mathrm{~s}$ from the start of trial). Pressing of the lever during the escape period (between delivery of the first and third shock) would retract the lever, cease additional shocks and initiate the safety signal. These trials were recorded as an "escape." If a rat did not press during the 10-s escape period, the lever retracted after the third shock and no safety signal was presented; these trials were recorded as "active avoidance failures." During this initial stage of avoidance training, rats were given daily sessions until they made $\sim 70 \%$ active avoidance and $<30 \%$ failures for at least three consecutive days before progressing to either the next phase of training (for the active/inhibitory avoidance experiments) or surgery, retraining and inactivation tests (for the single-cue active avoidance experiments).

Over the course of training, the shock current was initially set to $0.2 \mathrm{~mA}$ for all animals and individually titrated by increments of $0.05 \mathrm{~mA}$ over the course of training to reinforce avoidance behavior if performance did not improve or declined for over three consecutive days. Shock intensities (range 0.2-0.4 mA) were kept constant after surgery. Individual shock intensity for each session was the same for active and inhibitory avoidance trials.

\section{Active/inhibitory avoidance training}

After single-cue avoidance training, rats were then introduced to the active/inhibitory avoidance version of the task, with daily sessions consisting of 12 active and 12 inhibitory trials (Fig. 1A). Active trials were identical to those described for the initial phase of training and were signaled with a white noise. In contrast, inhibitory trials were associated with a $9-\mathrm{kHz}, 80-\mathrm{dB}$ tone. Importantly, previous work in our laboratory has shown that inactivation of either the PL or IL does not disrupt discrimination between white noise versus pure tones in rats performing an auditory conditional discrimination task (van Holstein and Floresco, 2020).

Each inhibitory trial started in a manner similar to active trials, in that the tone would play and the lever was inserted. However, during these trials, rats were required to withhold pressing of the lever for 15-s duration of the tone to avoid foot-shock. Pressing of the lever during this period immediately delivered a foot-shock. During these trials, the lever remained extended and tone stayed on for the entire 15-s period, so that animals could emit more than one lever press, each resulting in foot-shock. Trials where the animal pressed the lever at least once were recorded as "inhibitory avoidance failures" and number of presses was recorded for each trial. At the end of the 15-s period, the tone was silenced and the lever retracted. If no lever press was performed by the end of the 15-s trial, the same safety signal used in active trials was presented (i.e., 30-s light cue). Trials where no lever presses occurred were recorded as a "successful inhibitory avoidance." The latency to lever press in successful active avoidance, escapes, and inhibitory avoidance failure trials were recorded.

Initial training on this task used a blocked design, with blocks of trials presented as 12 active trials followed by 12 inhibitory trials. Over subsequent training sessions, the order of active/inhibitory blocks alternated each day. Rats were trained on this blocked version of the task until they achieved performance criteria of $\sim 70 \%$ successful trials in both active and inhibitory avoidance for at least three consecutive days. During this phase of training, a small proportion of rats that initially displayed criterion performance on active trials began to show a decline in performance over additional training (although performance on inhibitory trials remained high). In these situations, rats were given remedial sessions and trained on the previous phase of training (with only active avoidance trials) until criterion performance was reached again.

The final version of the task consisted of 12 active and 12 inhibitory trials as in earlier phases (Fig. 1A). However, active and inhibitory trials were now presented pseudorandomly (randomized in pairs). All other parameters remained identical to the previous stage of training and animals were trained on this intermixed task until they reached criteria of $>70 \%$ successful trials in both active and inhibitory trials for three consecutive days. During this phase of training, a small proportion of rats began performing poorly on the intermixed task design, and were given remedial sessions of the blocked design to rescue performance. After reaching criteria in this final portion of training, animals received surgical implantation of cannulas in PL or IL and were allowed to recover before being retrained to criterion for inactivation tests.

\section{Active/inhibitory reward-seeking}

A separate group of animals were trained on a task was designed to resemble the basic structure of the active/inhibitory avoidance task as closely as possible, except here animals had to either emit or withhold a lever-press response to obtain food reward (Fig. 1B). The task used the same auditory cues and interval ITI as in the avoidance task, and a 9-kHz tone or white noise auditory cue were associated with either active or inhibitory trials. Each trial started with lever insertion and initiation of one of the two auditory cues that instructed the animals to either press the lever within $15 \mathrm{~s}$ of its insertion or withhold pressing for $15 \mathrm{~s}$ to obtain a reward of two sucrose pellets (BioServ). On active trials, a lever press terminated the tone, retracted the lever, illuminated the stimulus 

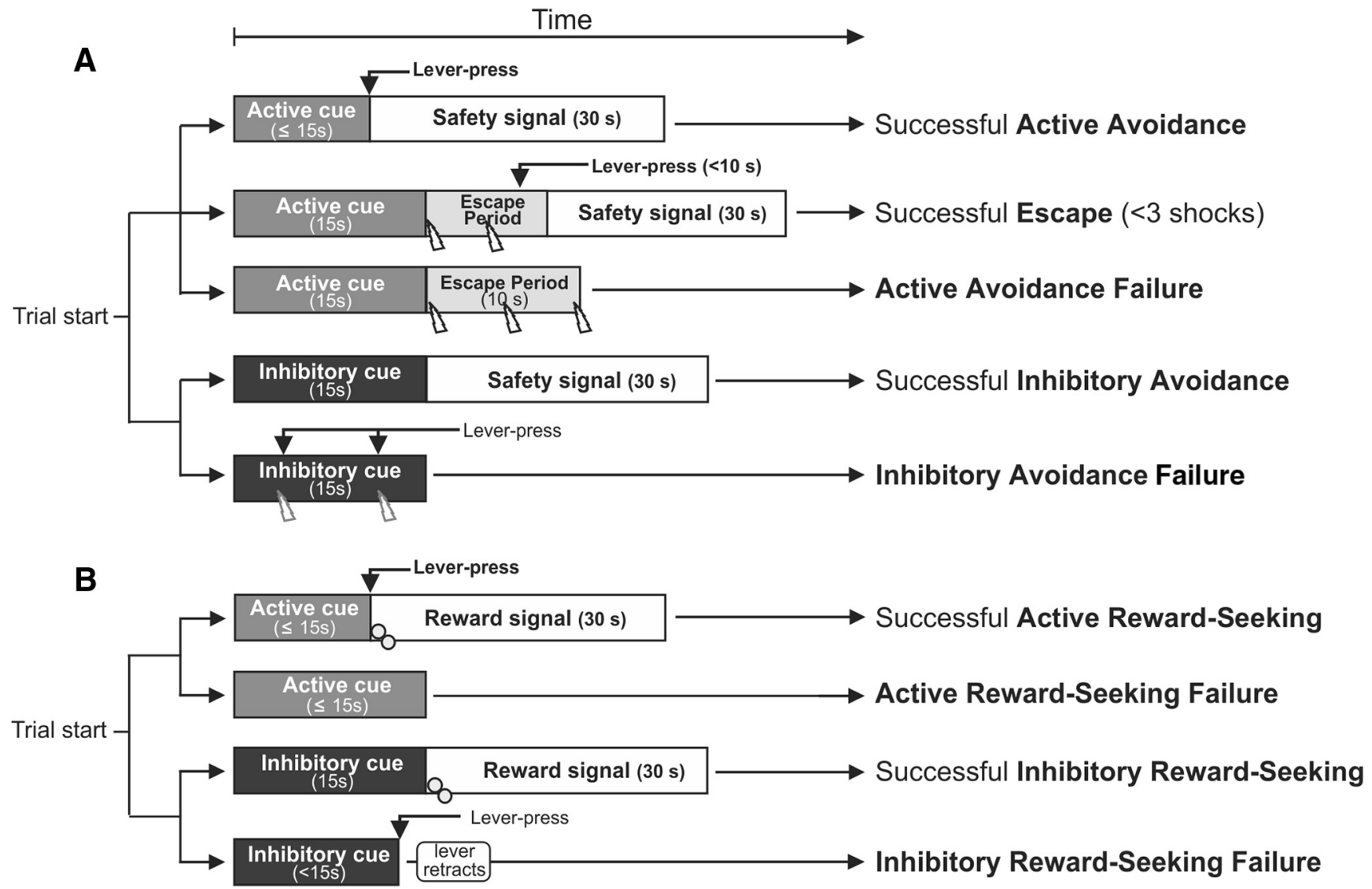

Figure 1. Task structure for active/inhibitory avoidance and reward-seeking. Potential scenarios and outcomes of each trial during $(\boldsymbol{A})$ active/inhibitory avoidance and $(\boldsymbol{B})$ active/inhibitory reward-seeking. Levers retracted after a successful avoidance/reward seeking response (active or inhibitory), escape or failures and were reinserted at the start of the next trial. Lightning bolt = shock delivery; gray circles = reward delivery. Time in seconds describes duration of cues, escape period, and safety/reward signal.

light/extinguished the house light for $30 \mathrm{~s}$ and delivered two pellets in the food port $3 \mathrm{~s}$ after the press. These responses were labeled "successful active reward-seeking" trials. Failure to press the lever during active trials resulted in lever retraction and the trial ended with no reward delivered, these trials were recorded as "active reward-seeking failure." On inhibitory trials, if a rat withheld pressing for the 15-s period, the tone terminated, the lever was retracted, the stimulus/house lights were illuminated/extinguished for $30 \mathrm{~s}$, two pellets were delivered $3 \mathrm{~s}$ later, and the trial was recorded as "successful inhibitory reward-seeking." In contrast, on inhibitory trials, if a rat pressed the lever during the 15-s tone presentation period, the lever retracted, no reward was delivered but the tone continued for the entire 15-s period and the trial was recorded as "inhibitory reward-seeking failure."

After receiving initial lever exposure, rats first received two to five sessions with 20 active trials and were then introduced to inhibitory trials in a blocked design like that used in avoidance training with 12 active and 12 inhibitory trials. During the initial stages of training, we observed that the number of successful active trials was substantially higher than inhibitory trials. To improve acquisition of inhibitory reward-seeking, on some days, rats received sessions of 20 exclusively inhibitory trials. After at least $21 \mathrm{~d}$ of training, animals were introduced to the intermixed presentation of active and inhibitory trials for $7 \mathrm{~d}$ or until criteria of $>50 \%$ successful active and inhibitory trials for four consecutive days. In practice, rats pressed the lever on active trials close to $100 \%$, whereas performance on the inhibitory trials was considerably poorer. Rats that received at least $7 \mathrm{~d}$ of training using an intermixed presentation of active/inhibitory trials and made at least $>40 \%$ successful inhibitory trials on saline (SAL) infusion days were included in our analysis. This permissive criterion was rationalized because of the difficulty encountered in training some animals to withhold lever pressing.

\section{Drugs and microinfusions}

Rats received their first of two counterbalanced microinfusion test days after displaying criterion performance for at least three consecutive days. On the day before the first infusion, rats received a mock infusion $10 \mathrm{~min}$ before the start of their daily training session, during which they were brought to the same room used for the following infusion days, obturators were removed, and a stainless-steel mock injector flush with the end of the guide cannula was inserted for $\sim 2 \mathrm{~min}$.

If performance remained stable on mock infusion day, the next day, a rat received its first of two infusions test day, otherwise, it was given two to three additional days of training, and mock procedure was repeated. On infusion days, animals received $0.4 \mu \mathrm{l}$ of either $0.9 \% \mathrm{SAL}$ or a solution containing $100 \mathrm{ng}$ each of the GABA-B agonist baclofen and the GABA-A agonist muscimol in SAL (BM; Tocris) through injectors inserted in the cannula guides. Previous studies have shown that infusion of these GABA agonists at a similar concentration and volume can induce dissociable effect on behavior when infused into regions separated by $\geq 1 \mathrm{~mm}$, such as the adjacent core and shell regions of the NAc (Floresco et al., 2018; Piantadosi et al., 2018). Of particular note, using identical surgical and microinfusion procedures, our laboratory has shown different and sometimes opposing effects of inactivation of the PL versus IL regions of the mPFC (St. Onge and Floresco, 2010; van Holstein and Floresco, 2020), including alterations in probabilistic reversal learning following inactivation of the PL, with no effect following IL inactivation (Dalton et al., 2016). This is in keeping with electrophysiological studies estimating the functional spread of GABA agonistinduced neural inactivations to be $\sim 1 \mathrm{~mm}$ (Martin and Ghez, 1999). Given these considerations, it is likely that the dissociable effects reported here were due to suppression of activity within the targeted mPFC region, and not due to spread to adjacent regions (e.g., backflow of GABA agonists from the IL into the PL). 


\section{A Active/inhibitory Avoidance}

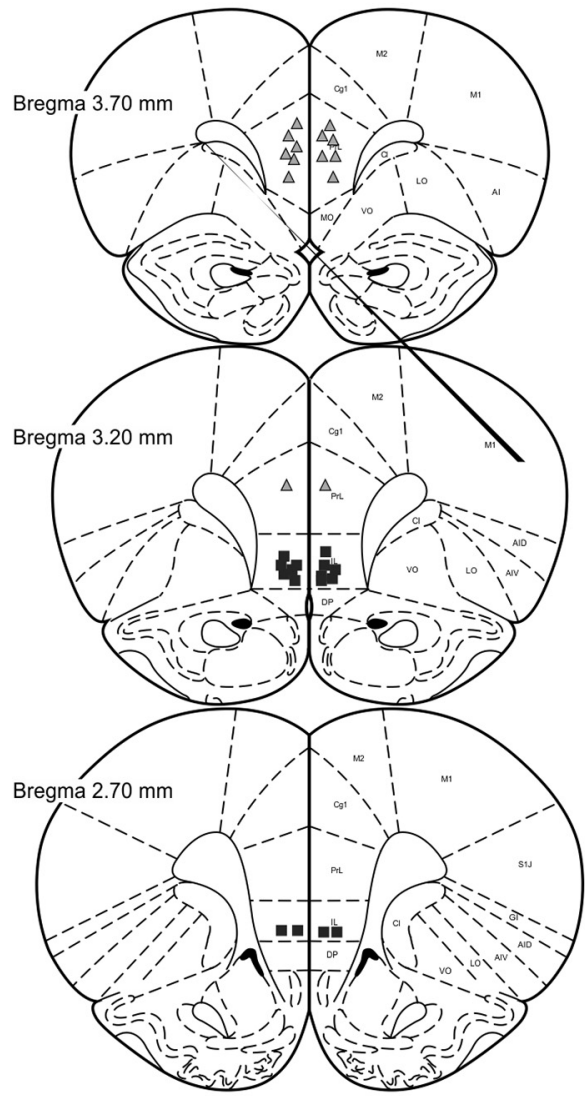

B Single-Cue Active Avoidance

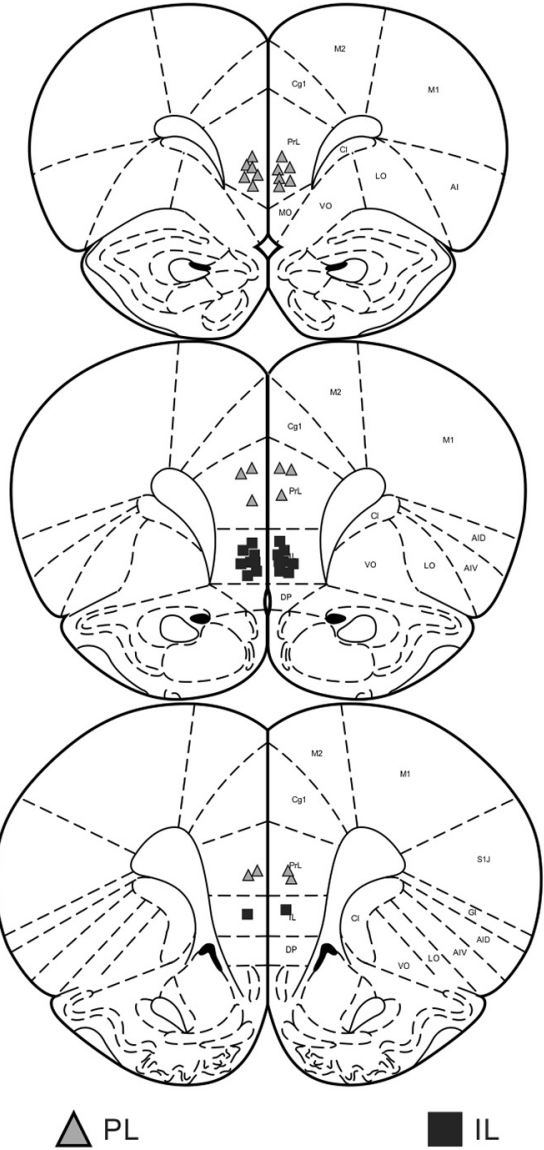

C

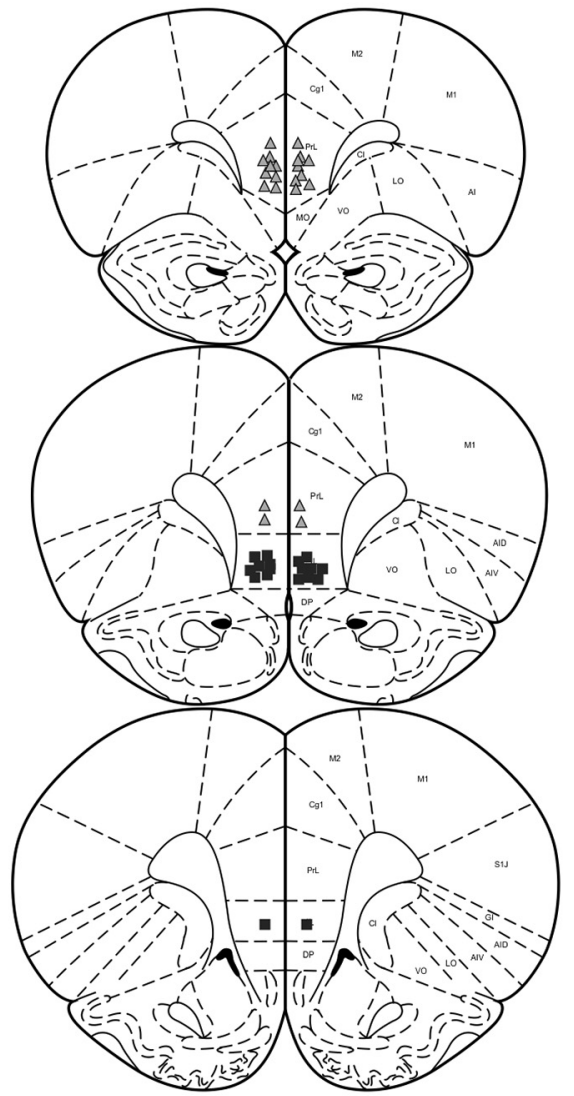

Figure 2. Histology. Schematic of coronal sections displaying the ventral extent of acceptable microinfusions in the PL (gray triangles) and IL (black squares). Placements are shown for rats in $(\boldsymbol{A})$ active/inhibitory avoidance, $(\boldsymbol{B})$ active avoidance, and $(\boldsymbol{C})$ active/inhibitory reward-seeking experiments.

Each infusion lasted $60 \mathrm{~s}$, and injectors were left in place for an additional $60 \mathrm{~s}$ for diffusion. Behavioral testing commenced $10 \mathrm{~min}$ after the end of the infusion. After the first infusion test, rats were retrained for at least $2 \mathrm{~d}$ until they again displayed criterion performance, after which they received their second counterbalanced infusion.

Histology

After the completion of testing, rats were anaesthetized with $4 \%$ isofluorane and euthanized with $\mathrm{CO}_{2}$. Brains were removed and fixed in a $4 \%$ formalin buffered SAL solution and stored until sectioning. Using a cryostat, $50-\mu \mathrm{m}$ sections were collected and mounted on glass slides. All brains were Nissl stained with cresyl violet and acceptable cannula placements are reported in Figure 2. Data from animals whose placement did not reside within the intended region were removed from analysis.

Experimental design and statistical analyses

For all tasks and trial types, successful trials, failures, and escapes were converted to a percentage of total trials of each trial type (i.e., active or inhibitory). Percentage of successful active and inhibitory trials were analyzed separately for each brain region using two-way repeated measures ANOVAs with treatment (SAL vs inactivation) and trial type (active or inhibitory) as two within-subject factors. The percentage of escapes, the number of shocks received, and response latencies were each analyzed with separate one-way repeated measures ANOVAs with Treatment as the within-subject factor. In situations where a rat did not make any response on active or inhibitory trials during a test session, its latency values were set to $15 \mathrm{~s}$ (the maximum time allotted). Locomotor data were converted into beam-breaks/min, and these values were analyzed separately for each region and task group using one-way repeated measures ANOVAs. Shock intensities used during testing measured in $\mathrm{mA}$ for each animal were analyzed separately for the active/inhibitory and the active avoidance tasks using one-way ANOVAs to compare groups assigned to either brain region.

Supplementary analyses of how treatments affected performance over the course of a test session were conducted by grouping the percentage of successful trials were into blocks of two trials and analyzed via two-way repeated measures ANOVA with treatment and trial block as within-subject factors.

Lastly, learning rates for different types of trials (active vs inhibitory) of each task were calculated by counting the number of training days each rat required to display criterion performance for two consecutive days. For each type of response, the data were analyzed separately with one-way ANOVAs. Prescribed $\alpha$ level for all analyses was 0.05. Data are reported as mean \pm SEM.

\section{Results}

\section{Active/inhibitory avoidance}

Initial learning

All rats $(n=26)$ successfully learned the initial active avoidance contingency, taking between 6 and 27 sessions to reach criterion for an average of $11.2 \pm 1.1$ sessions. On the final day of their active avoidance training, rats performed $78.1 \pm 3 \%$ active avoidance responses, $5.4 \pm 1 \%$ escapes, and $16.5 \pm 3 \%$ failures. Rats then progressed to the blocked design, during which they received active avoidance followed by inhibitory avoidance trials and vice versa on alternate days. Out of the initial 26 animals, 


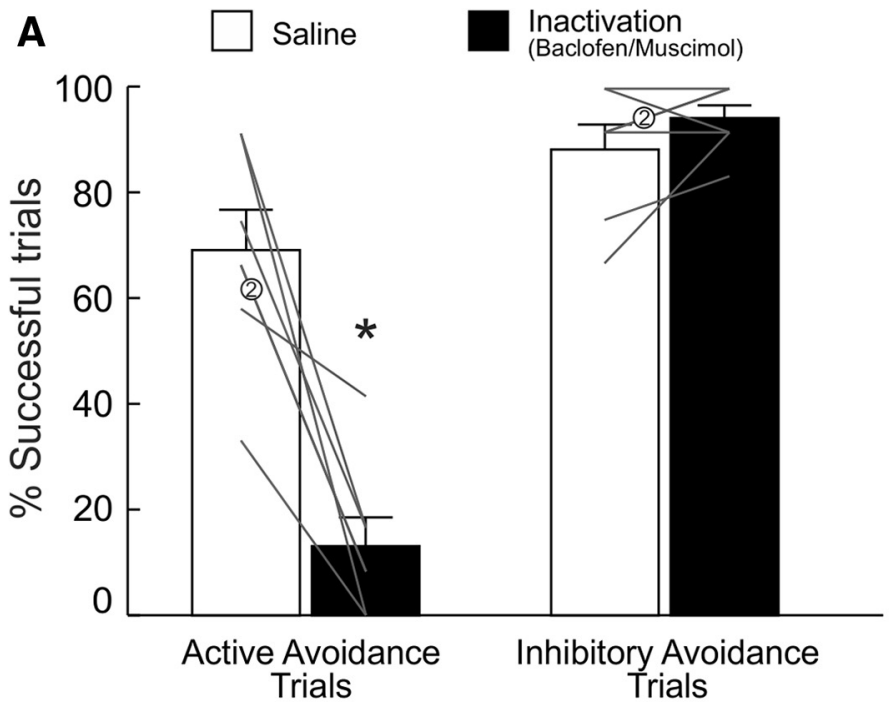

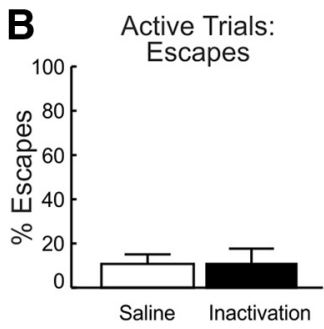

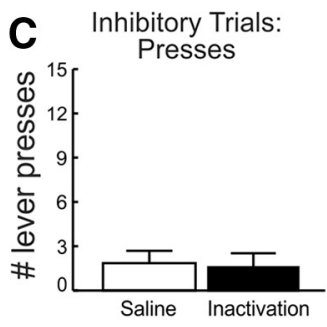

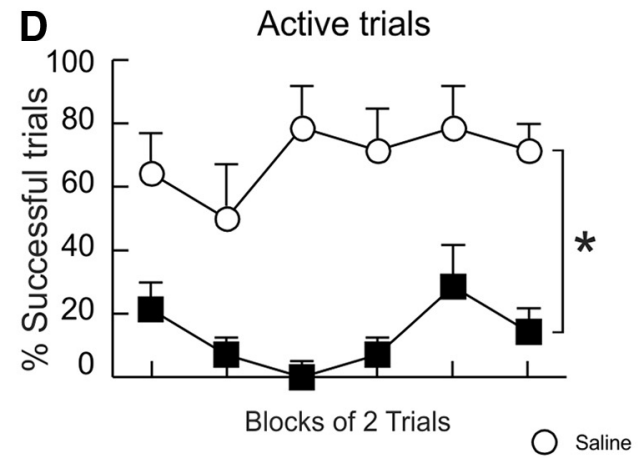

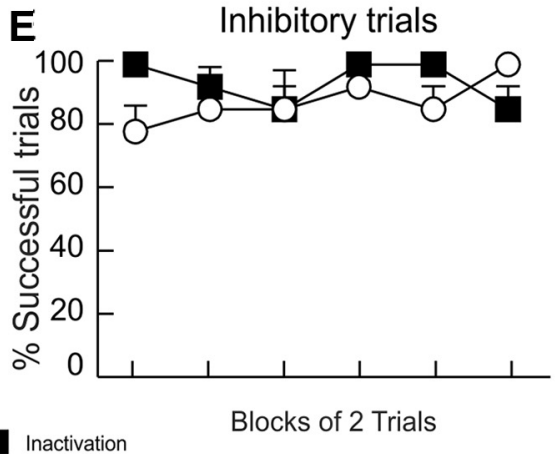

Figure 3. PL inactivation impaired active, but not inhibitory, avoidance performance. $A$, PL inactivation decreased the mean percentage of successful active (left) avoidance trials but had no effect on inhibitory trials (right). $\boldsymbol{B}$, Escape responses were unaltered by PL inactivation. $\boldsymbol{C}$, The total number of lever presses on inhibitory avoidance trials were also unaltered by PL inactivation. $\boldsymbol{D}, \boldsymbol{E}$, Percentage of successful active and inhibitory avoidance responses following SAL and inactivation treatments, partitioned over blocks of two trials. The effects of PL inactivation on active avoidance were apparent from the beginning of the session. For this and all other figures, error bars represent SEM, gray lines represent data from individual animals, numbers denote number of overlapping data points for a particular line, and asterisks denotes $p<0.05$ between the SAL versus inactivation conditions $(n=7)$.

$n=23$ reached criterion on this blocked design after $18 \pm 1.8$ sessions (range of 7-35 sessions). On the final day of training, rats performed $84.8 \pm 3 \%$ active avoidance responses, $4.5 \pm 2 \%$ escapes, $10.6 \pm 2 \%$ active avoidance failures, and $82.4 \pm 2 \%$ successful inhibitory avoidance trials.

Of the 23 rats that progressed on to the last stage of intermixed active/inhibitory avoidance training, 21 rats reached criterion taking on average $11.9 \pm 1.1$ sessions (range 3-27 sessions). On their last day of intermixed avoidance training, animals that successfully reached criterion made $84.7 \pm 4 \%$ successful active avoidance responses, $5.1 \pm 1.8 \%$ escapes, $10.2 \pm 3 \%$ active avoidance failures, and $88.4 \pm 2 \%$ successful inhibitory avoidance trials. Data from the five animals that did not reach criterion were not included in the final analysis.

Of the 21 rats that achieved the final task criteria and were implanted with guide cannula into either the PL or IL, six were excluded from the final analysis due to either cannula placements outside of the region of interest $(n=4)$, unexpected mortality following surgery $(n=1)$, or failure to recover performance following surgery $(n=1)$. This resulted in final sample sizes of seven and eight for the PL and IL groups, respectively. The mean shock intensity for PL group was $0.32 \pm 0.03 \mathrm{~mA}$ (range 0.30$0.35 \mathrm{~mA}$ ), while for the IL it was $0.30 \pm 0.04 \mathrm{~mA}$ (range $0.25-$ $0.35 \mathrm{~mA}$ ). These intensities did not differ significantly across groups $\left(F_{(1,13)}=1.56, p=0.23\right)$.

\section{$P L$ inactivation}

Analysis of successful active and inhibitory trials exposed a significant main effect of treatment $\left(F_{(1,6)}=24.39, p<0.01\right)$ and, in particular, a treatment $\times$ trial type interaction $\left(F_{(1,6)}=36.37\right.$, $p<0.001)$, suggesting that PL inactivation differentially affected performance on active versus inhibitory trials (Fig. $3 A$ ). Interestingly, this analysis revealed a significant main effect of trial type $\left(F_{(1,6)}=61.71, p<0.001\right)$, indicating that animals performed significantly better on inhibitory versus active avoidance trials across treatment conditions.

Simple main effects analyses confirmed that PL inactivation markedly reduced the percentage of successful active avoidance responses $\left(F_{(1,6)}=59.44, p<0.001\right.$; Fig. $3 A$, left $)$. Escapes on active trials were comparatively low and did not differ across treatments $\left(F_{(1,6)}=0, p=1\right.$; Fig. $\left.3 B\right)$. In contrast, PL inactivation did not alter performance on inhibitory trials $\left(F_{(1,6)}=0.67\right.$, $p=0.44$; Fig. $3 A$, right) nor did it alter the number of lever presses on these trials $\left(F_{(1,6)}=0.22, p=0.654\right.$; Fig. $\left.3 C\right)$. Reflecting the impairment on active trials, rats received more foot-shocks on PL inactivation days $\left(F_{(1,6)}=48.59, p<0.001\right.$; Table 1$)$.

$\mathrm{PL}$ inactivation tended to increase response latencies on active trials but this effect did not achieve statistical significance $\left(F_{(1,6)}=4.10, p=0.089\right.$; Table 1$)$. Latency to press the lever in inhibitory trials was not affected by PL inactivation $\left(F_{(1,6)}=0.14, p=0.73\right.$; Table 1$)$. Locomotion (beam breaks/ 
Table 1. Performance measures during active/inhibitory avoidance, active avoidance, and active/inhibitory reward-seeking tasks

\begin{tabular}{|c|c|c|c|c|}
\hline & PL (control) & PL (inactivation) & IL (control) & IL (inactivation) \\
\hline \multicolumn{5}{|l|}{ Active/inhibitory avoidance } \\
\hline Active avoidance latency (s) & $5.75 \pm 0.8$ & $9.49 \pm 1.9$ & $6.11 \pm 1.3$ & $7.6 \pm 1.1$ \\
\hline Inhibitory avoidance failure latency (s) & $9.21 \pm 1.87$ & $8.34 \pm 2.4$ & $7.6 \pm 2.4$ & $6.78 \pm 1.1$ \\
\hline Locomotion (beam breaks/min) & $15.8 \pm 1.7$ & $16.8 \pm 3.3$ & $19.6 \pm 2.0$ & $29.5 \pm 5.1$ \\
\hline \multicolumn{5}{|l|}{ Active avoidance } \\
\hline Avoidance latency (s) & $5.04 \pm 0.4$ & $4.56 \pm 0.5$ & $5.57 \pm 0.41$ & $5.59 \pm 0.54$ \\
\hline \multicolumn{5}{|l|}{ Active/inhibitory reward-seeking } \\
\hline Active response latency (s) & $1.01 \pm 0.1$ & $2.34 \pm 0.6^{*}$ & $1.41 \pm 0.3$ & $1.34 \pm 0.2$ \\
\hline Inhibitory failure latency (s) & $4.64 \pm 1.4$ & $2.9 \pm 0.6$ & $3.9 \pm 1.2$ & $3.21 \pm 0.6$ \\
\hline Rewards received & $36.7 \pm 1.4$ & $25.1 \pm 1.2 * * *$ & $38.4 \pm 1.2$ & $26 \pm 0.8^{* * *}$ \\
\hline Locomotion (beam breaks/min) & $18.9 \pm 3.3$ & $21.3 \pm 4.2$ & $21.1 \pm 2.8$ & $32.7 \pm 5.4$ \\
\hline
\end{tabular}

Values displayed are mean $\pm \mathrm{SEM} ;{ }^{*} p<0.01,{ }^{* *} p<0.001,{ }^{* * *} p<0.0001$.

min) across sessions did not differ across treatment conditions $\left(F_{(1,6)}=0.22, p=0.66\right.$; Table 1$)$.

Supplementary analyses examined how PL inactivation altered performance over the course of the test session. This analysis revealed that the impairment of active avoidance induced by inactivation of this region was apparent from the beginning and persisted throughout the test session (Fig. 3D). Accordingly, analysis of active avoidance performance over a session yielded a main effect of treatment $\left(F_{(1,6)}=35.43, p=0.001\right)$, but no treatment $\times$ block interaction $\left(F_{(5,30)}=0.86, p=0.52\right)$. A similar analysis conducted on data from inhibitory trials also found no effects of treatment (interaction: $F_{(5,30)}=1, p=0.44$; treatment: $F_{(1,6)}=2.27, p=0.18$; Fig. $\left.3 E\right)$. Collectively, these data show that PL inactivation selectively impaired the ability to initiate instrumental responses to avoid foot-shock when signaled by discriminative cues, without affecting inhibitory avoidance. Thus, activity in this region is necessary for active defensive behaviors in response to learned cues in aversive contexts.

\section{IL inactivation}

In contrast to the more selective effects of PL inactivation, similar treatments in the IL in a separate group of rats impaired performance on both active and inhibitory trials (Fig. 4A). The ANOVA on these data yielded a significant main effect of treatment $\left(F_{(1,7)}=23.25, p=0.002\right)$ but no main effect of trial type $\left(F_{(1,7)}=1.33, p=0.29\right)$ nor treatment $\times$ trial type interaction $\left(F_{(1,7)}=0.72, p=0.45\right)$. The impairment on active avoidance trials was not accompanied by a change in the proportion of escapes $\left(F_{(1,7)}=0.88, p=0.38\right.$; Fig. $\left.4 B\right)$. On the other hand, the impairment on inhibitory trials was associated with an increased number of total lever presses on these trials $\left(F_{(1,7)}=7.22\right.$, $p=0.03$; Fig. $4 C$ ).

IL inactivation tended to slow latencies to press on active trials and this effect approached statistical significance $\left(F_{(1,7)}=\right.$ 4.57, $p=0.07$; Table 1) but did not alter latencies to press on inhibitory trial failures $\left(F_{(1,7)}=0.08, p=0.79\right.$; Table 1$)$. In accordance with the broad disruption in performance, rats received more shocks following IL inactivation $\left(F_{(1,7)}=46.49, p<0.001\right.$; Table 1). Rats, on average, made more beam breaks per minute following IL inactivation but this measure of locomotion did not achieve statistical significance $\left(F_{(1,7)}=3.90, p=0.09\right.$; Table 1$)$.

Analysis of active and inhibitory avoidance performance over blocks of two trials confirmed effect of Treatment remained significant for both active $\left(F_{(1,7)}=29.87, p<0.001\right)$ and inhibitory trials $\left(F_{(1,7)}=7.41, p=0.03\right)$. This two-way
ANOVA also demonstrated the effect of IL inactivation was apparent at the start of the session and remained consistent over trial blocks, as analysis of these data failed to yield significant treatment $\times$ block interaction and effect of block on either active (both $F_{(5,35)}<1.14$, both $p s>0.36$; Fig. $4 D$ ) or inhibitory trials (both $\mathrm{Fs}_{(5,35)}<0.94$, both $p s>0.47$; Fig. $4 E$ ). These data implicate IL in the use of discriminative stimuli to appropriately avoid aversive consequences, by both facilitating and inhibiting actions to avoid punishment.

\section{Single-cue active avoidance}

Pharmacological inactivation of both the IL and PL impaired active avoidance when animals were required to discriminate between cues signaling active and inhibitory trials. Following from these results, we investigated the involvement of these prefrontal regions in absence of a discriminatory component using a simpler task during which a single cue signaled lever-press response could avoid foot-shock.

\section{Initial learning}

A separate group of rats initially comprised of 31 animals, with 29 of these achieving criterion performance. Rats received on average $14 \pm 1$ sessions before reaching criterion and undergoing surgery (range 4-26 sessions). On their last day of active avoidance training, average performance was $86 \pm 3 \%$ active avoidance responses, $2.8 \pm 0.9 \%$ escapes, and $11.2 \pm 3 \%$ failures.

Of the 29 rats that achieved the final criterion performance and were implanted with guide cannula, nine were excluded from the final analysis due to either cannula placements outside of the region of interest $(n=6)$ or unexpected mortality following surgery $(n=3)$. This resulted in a final $n=10$ for each PL and IL group. The mean shock intensity were comparable across the PL $(0.29 \pm 0.05 \mathrm{~mA}$; range $0.20-0.35 \mathrm{~mA})$ and IL groups $(0.30 \pm$ $0.06 \mathrm{~mA}$; range $\left.0.20-0.35 \mathrm{~mA} ; F_{(1,18)}=0.16, p=0.70\right)$.

\section{PL inactivation}

In stark contrast to what observed following PL inactivation on the active/inhibitory avoidance task, similar treatment did not alter performance on the single-cue active avoidance task, although these rats received considerably less training than those in the active/inhibitory avoidance experiment. Analyses of these data revealed no significant differences in the proportion of successful active avoidance responses across treatments $\left(F_{(1,9)}=\right.$ $0.21, p=0.66$; Fig. $5 A$, left $)$, escapes $\left(F_{(1,9)}=0.05, p=0.83\right.$; Fig. $5 B$, left), number of foot-shocks $\left(F_{(1,9)}=0.18, p=0.68\right.$; Table 1$)$, 

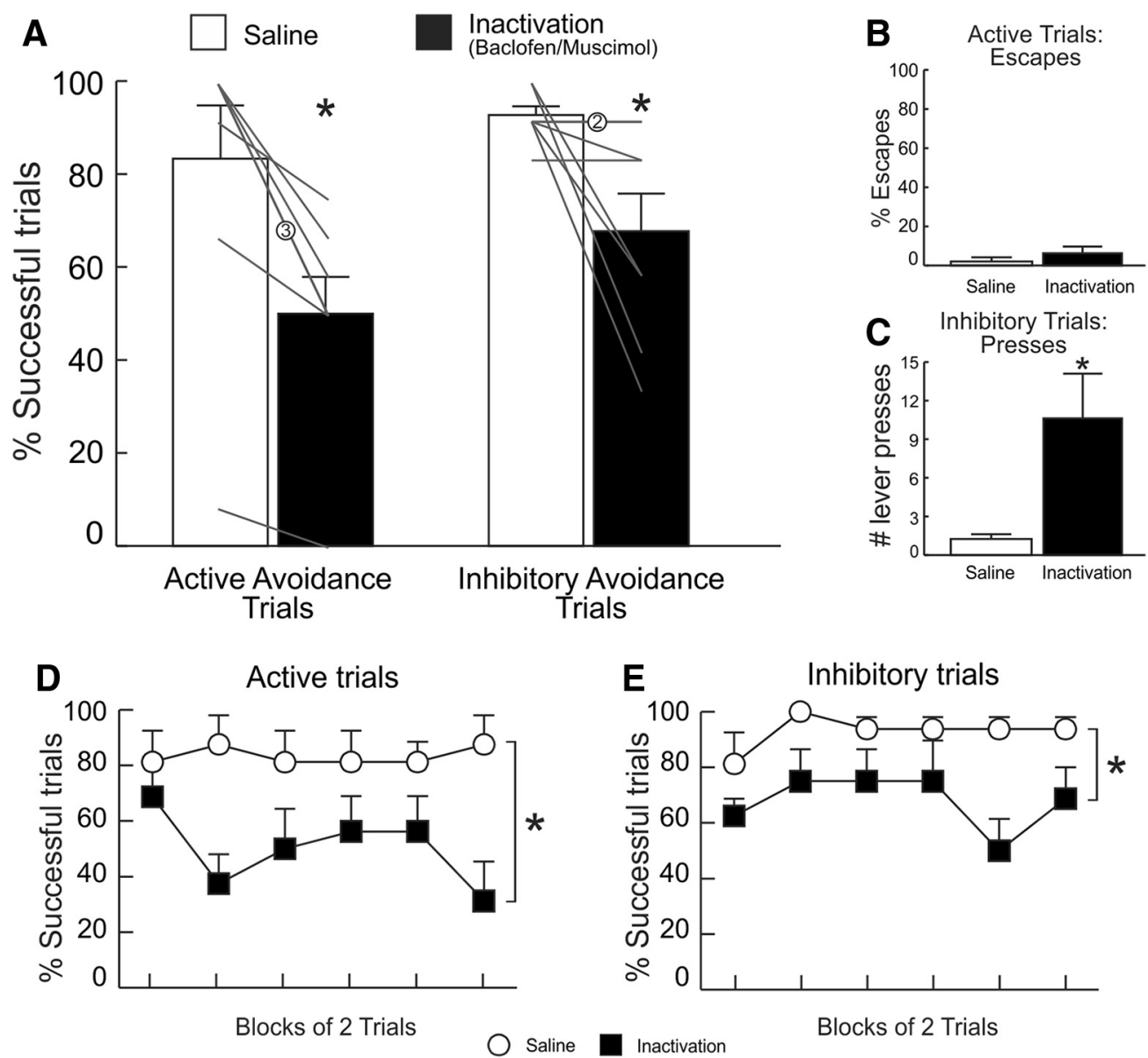

Figure 4. IL inactivation impaired active and inhibitory avoidance performance. $A$, IL inactivation decreased the percentage of successful active (left) and inhibitory (right) avoidance trials. $\boldsymbol{B}$, IL inactivation did not alter escape responses. $\boldsymbol{C}$, IL inactivation also increased the total number of lever presses on inhibitory avoidance trials. $\boldsymbol{D}, \boldsymbol{E}$, Percentage of successful, active and inhibitory avoidance responses partitioned over blocks of two trials $(n=8)$.

or response latencies $\left(F_{(1,9)}=0.63, p=0.45\right.$; Table 1$)$. Locomotion was also unchanged across conditions $\left(F_{(1,9)}=1.80\right.$, $p=0.21$; Table 1). Similarly, analysis of active avoidance trials across blocks of two trials (Fig. $5 C$ ) did not yield a significant main effect of treatment $\left(F_{(1,9)}=0.21, p=0.66\right)$ or treatment $\times$ block interaction $\left(F_{(9,81)}=1.05, p=0.41\right)$. These data indicate that PL activity does not appear to be essential to facilitate instrumental avoidance in response to a singular warning stimulus when allocation of behavior is unambiguous.

\section{IL inactivation}

In contrast to the lack of effect of PL inactivation on active avoidance, IL inactivation impaired single-cue active avoidance in a manner similar to that observed during active/inhibitory avoidance (Fig. 5A, right). Analysis of these data revealed significant reduction in active avoidance responses after inactivation treatments relative to SAL $\left(F_{(1,9)}=19.40, p<0.01\right)$ but no effect on escape behavior $\left(F_{(1,9)}=2.50, p=0.15\right.$; Fig. $5 B$, right $)$. Accordingly, rats received significantly more foot-shocks following IL inactivation $\left(F_{(1,9)}=13.12, p<0.01\right.$; Table 1$)$. IL inactivation did not affect latency to lever press $\left(F_{(1,9)}=0.001, p=0.97\right.$; Table 1), and again, locomotor counts were increased by these treatments, but the effect did not reach statistical significance $\left(F_{(1,9)}=3.89, p=0.08\right.$; Table 1$)$. When analyzed across two-trial blocks (Fig. 5D), performance did not yield significant main effect of block $\left(F_{(9,81)}=1.05, p=0.41\right)$ and no treatment $\times$ block interaction was found $\left(F_{(9,81)}=0.75, p=0.67\right)$, while treatment remained significant $\left(F_{(1,9)}=19.40, p<0.01\right)$. These data suggest that the IL facilitates active instrumental behaviors intended to avoid aversive events, even in circumstances that do not require cue discrimination and flexible responding.

\section{Active/inhibitory reward-seeking}

Our studies to this point established that the PL and IL are differentially recruited in either encouraging or withholding actions to avoid aversive outcomes. This prompted us to examine how these cortical regions may contribute to behavioral control in a similar situation that required either promotion or suppression of instrumental action, but when the valence of the outcome was positive (appetitive), rather than negative (aversive). Here, we probed how inactivation of these regions affected cued active and inhibitory goal-directed behaviors when making or withholding a response delivered sucrose reward.

\section{Initial learning}

A group of 30 rats, separate from those used in the active/inhibitory avoidance experiment, were trained on the rewarded active/ inhibitory contingency. They all received two to five initial sessions solely comprising of active trials for an average of $3.5 \pm 0.3$ sessions. In all subsequent training sessions, rats were presented with inhibitory trials, either with or without active trials. Animals received on average $15.1 \pm 1$ sessions of only inhibitory trials (range 8-25) and $17.7 \pm 0.8$ sessions (range 25-11) of active trials followed by inhibitory trials or vice versa, during training 

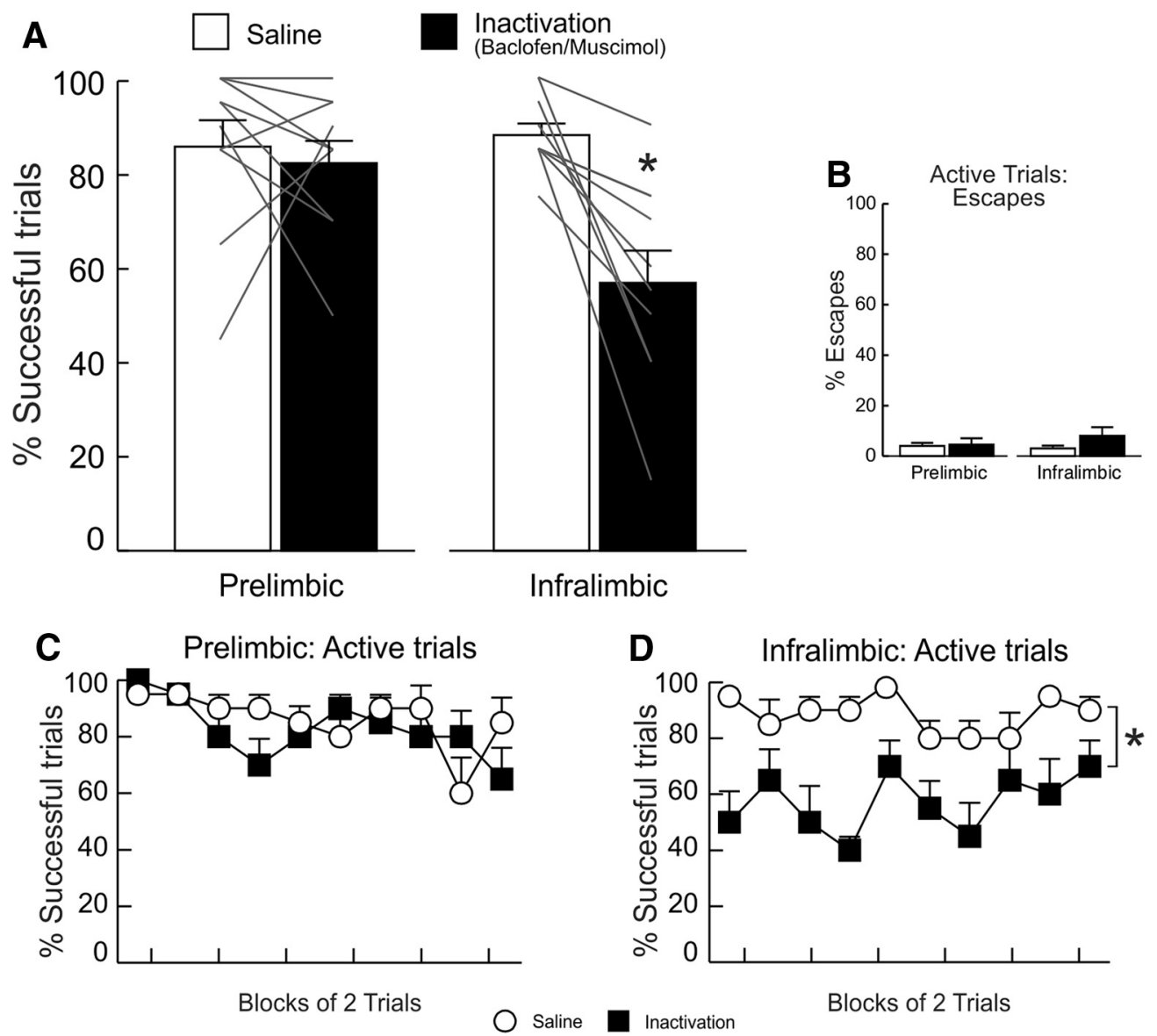

Figure 5. IL, but not PL, inactivation impaired single-cue active avoidance performance. $\boldsymbol{A}$, PL inactivation (left) had no effect on active avoidance whereas IL inactivation (right) decreased the percentage of successful active avoidance trials. $\boldsymbol{B}$, Inactivation of either region did not affect percentage of escape responses. $\boldsymbol{C}$, Percentage of successful active avoidance responses, partitioned over blocks of two trials, following PL treatments. $\boldsymbol{D}$, Percentage of successful active avoidance responses, partitioned over blocks of two trials following SAL and IL inactivation treatments (PL $n=10 ; \mathrm{IL} n=10)$.

with the blocked design protocol. On their last session of inhibitory training before testing, animals scored on average 57.3.5 \pm $4.8 \%$ successful inhibitory trials, while during the last session of active/inhibitory blocked trials rats performed $98.1 \pm 2.0 \%$ active responses and $52.8 \pm 5.6 \%$ successful inhibitory trials.

The final stage of intermixed presentation of active and inhibitory trials lasted on average $9.9 \pm 1.1$ sessions. On their last day of intermixed training, five rats failed to reach performance criterion. The remaining 25 rats all performed 100\% successful active responses and an average of $63.7 \pm 3.4 \%$ successful inhibitory trials.

Of the 25 rats that achieved criterion performance and received microinfusion tests, five were excluded from the final analysis due to cannula placements outside of the region of interest. This resulted in final sample sizes of 11 for PL and nine for the IL groups, respectively.

\section{$P L$ inactivation}

Inactivation of PL during this appetitive task had a diametrically opposite effect to that observed on the avoidance task, in that it markedly impaired performance on inhibitory trials, while sparing performance on active trials (Fig. 6A). A two-way ANOVA on the performance data revealed significant effects for both treatment $\left(F_{(1,10)}=62.87, p<0.0001\right)$ and, more pertinently, a treatment $\times$ trial type interaction $\left(F_{(1,10)}=18.15, p<0.01\right)$. Subsequent partitioning of the interaction with one-way ANOVAs revealed no significant change in performance during active trials following PL inactivation versus control treatments $\left(F_{(1,10)}=3.65\right.$, $p=0.09$; Fig. $6 A$, left). Notably, under control conditions, all rats in this experiment displayed a $100 \%$ success rate on active trials. Accordingly, to accommodate for the lack of variance in this condition, a supplemental non-parametric analysis on the active trials data were conducted using Wilcoxon signed-rank test. This revealed that the modest reduction in active reward seeking response following PL inactivation was statistically significant $(p=0.02)$. On the other hand, the ability to withhold responding to obtain a reward on inhibitory trials was much more markedly impaired by PL inactivation $\left(F_{(1,10)}=62.98, p<0.0001\right.$; Fig. 6 A, right $)$. Accordingly, rats also received significantly fewer reward pellets following inactivation versus SAL treatments $\left(F_{(1,10)}=47.96, p<0.0001\right.$; Table 1$)$.

Notably, the ANOVA of the performance data also yielded a significant main effect of trial type $\left(F_{(1,10)}=92.95, p<0.0001\right)$. Examination of percentage successful trials plotted in Figure $6 \mathrm{~A}$ indicates that animals performed better on active versus inhibitory trials. To investigate this effect, we compared the mean scores for active and inhibitory trials on drug-free baseline days that preceded the test days. Our analysis exposed a significant difference between baseline performance for active and inhibitory avoidance $\left(F_{(1,10)}=39.53, p<0.0001\right)$ in that rats found it considerably more difficult to appropriately inhibit lever pressing $(60.6 \pm 6 \%$ successful trials at baseline) compared with making these responses in the pursuit of sucrose reward (100 $\pm 0 \%)$. These results were opposite to what observed for aversively- 

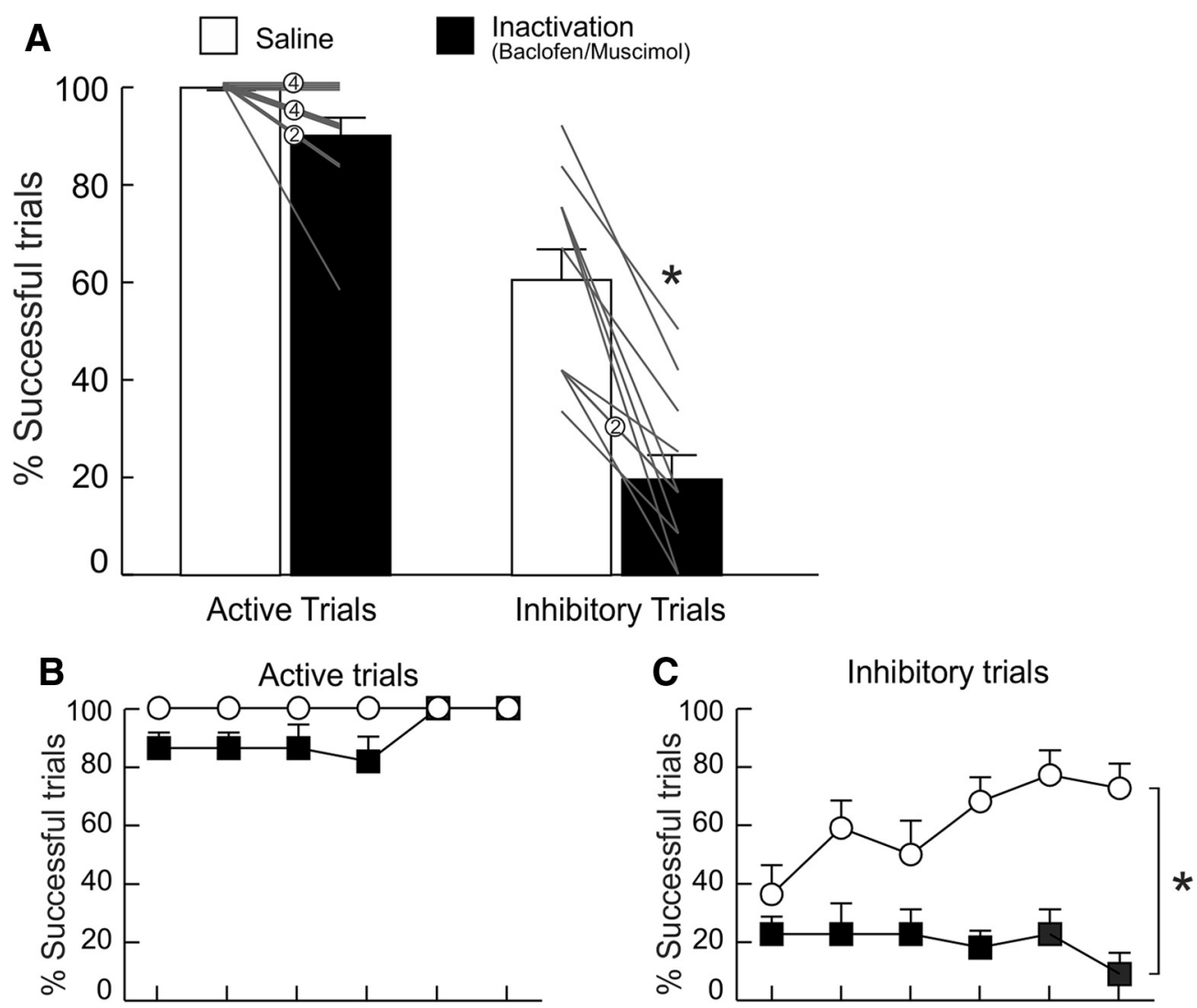

Blocks of 2 Trials

Inactivation

Blocks of 2 Trials

Figure 6. PL inactivation impaired inhibitory, but not active, reward-seeking. $A, P L$ inactivation did not alter lever pressing for reward on active trials (left) but impaired performance on inhibitory trials, when rats had to withhold pressing for $15 \mathrm{~s}$ to obtain reward (right). $\boldsymbol{B}, \boldsymbol{C}$, Percentage of successful active and inhibitory reward-seeking responses partitioned over blocks of two trials $(n=11)$

motivated active/inhibitory trials, for which performance tended to be better on inhibitory versus active trials.

PL inactivation increased response latencies on active trials $\left(F_{(1,10)}=6.81, p=0.03\right.$; Table 1$)$. On the other hand, the reduction in successful inhibitory trials was not associated by a changes in press latencies $\left(F_{(1,10)}=2.55, p=0.14\right.$; Table 1$)$. These treatments did not alter locomotion $\left(F_{(1,10)}=0.19, p=0.67\right.$; Table 1).

A two-way ANOVA conducted on data from active performance over blocks of two trials yielded no treatment $\times$ block interaction $\left(F_{(5,50)}=1.42, p=0.23\right)$ and no effect of block $\left(F_{(5,50)}=\right.$ $1.42, p=0.23)$ but exposed a significant treatment effect $\left(F_{(1,10)}=\right.$ $7.10, p=0.02$ ) that was driven by a slight reduction in performance early in sessions (Fig. $6 \mathrm{~B}$ ). For inhibitory trials, inspection of Figure $6 C$ shows that performance under control conditions was initially poor and then improved over the course of the session, whereas it remained poor throughout the session following inactivation treatments. However, the analysis of these data only yielded a significant main effect of treatment $\left(F_{(1,10)}=50.10\right.$, $p<0.0001)$ but no main effect of block $\left(F_{(5,50)}=1.06, p=0.39\right)$ or treatment $\times$ block interaction $\left(F_{(5,50)}=1.68, p=0.16\right)$. Collectively, these data show that the PL plays a more prominent role in using discriminative cues to suppress actions to obtain rewards, which contrasts to its role in facilitating actions during active/inhibitory avoidance.

\section{IL inactivation}

Like inactivation of the PL, similar treatment of the IL had different effects on active and inhibitory performance (Fig. 7A).
Analysis of the performance data revealed a significant main effects of treatment $\left(F_{(1,8)}=169.81, p<0.0001\right)$ and treatment $\times$ trial type interaction $\left(F_{(1,8)}=140.42, p<0.0001\right)$. The analysis also yielded significant main effect of trial type $\left(F_{(1,8)}=209.16\right.$, $p<0.0001)$, again indicative of rats showing poorer performance on inhibitory versus active trials. Simple main effects analysis further confirmed that, in contrast to the avoidance task, IL inactivation did not alter performance on active trials $\left(F_{(1,8)}=0.08\right.$, $p=0.79$; Fig. $7 A$, left). On the other hand, suppression of instrumental action on inhibitory trials was again impaired following IL inactivation $\left(F_{(1,8)}=271.70, p<0.0001\right.$; Fig. $7 A$, right $)$. When comparing amount of pellets received, ANOVA revealed rats received fewer reward after IL inactivation test days versus control treatments $\left(F_{(1,8)}=142.55, p<0.0001\right.$; Table 1$)$.

No significant differences in latency were observed during either active $\left(F_{(1,8)}=0.031, p=0.86\right.$; Table 1$)$ or inhibitory trials $\left(F_{(1,8)}=0.75, p=0.41\right.$; Table 1$)$. Once again, IL inactivation tended to increase in locomotor activity, but this measure only approached statistical significance $\left(F_{(1,8)}=5.03, p=0.055\right.$; Table 1).

Analysis of performance on active trials over blocks found no significant effects of treatment, block, or interaction between these factors (all $F s<1.0$, all $p s>0.35$; Fig. $7 B$ ). Analysis of inhibitory trials across two-trial blocks (Fig. $7 C$ ) yielded a main effect of treatment $\left(F_{(1,8)}=160, p<0.0001\right)$ with no effects of block $\left(F_{(5,40)}=1.18, p=0.34\right)$ or treatment $\times$ block interaction $\left(F_{(5,40)}=0.87, p=0.51\right)$. Taken together, these findings suggest that under these conditions, activity within the PL or IL plays a 

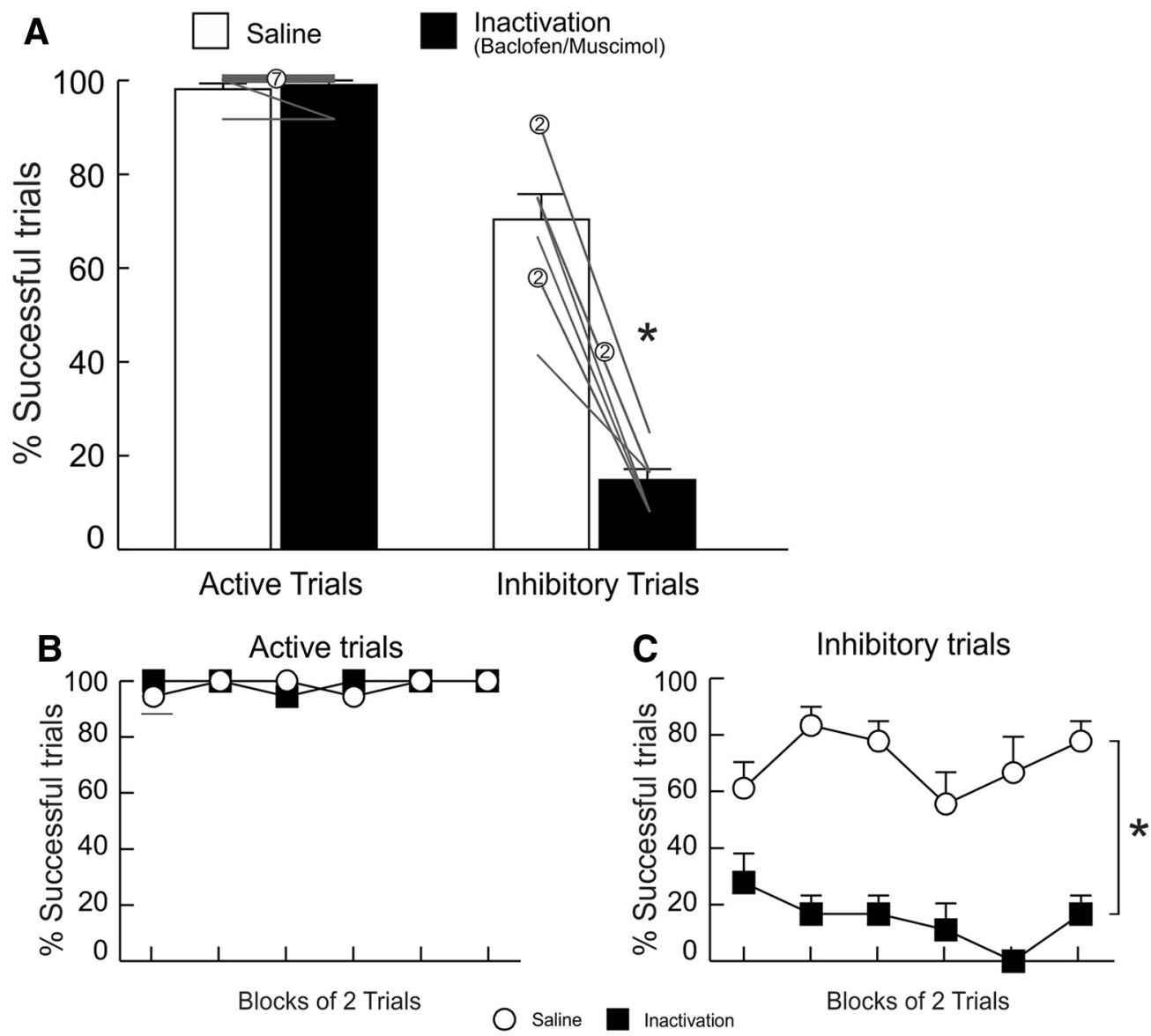

Figure 7. IL inactivation impaired inhibitory, but not active, reward-seeking performance. A, IL inactivation had no effect on active trials (left) but, like PL inactivation, decreased the percentage of successful inhibitory trials (right). $\boldsymbol{B}, \boldsymbol{C}$, Percentage of successful, active and inhibitory reward-seeking responses partitioned over blocks of two trials $(n=9)$.

minimal role in prompting instrumental actions triggered by discriminative cues indicating they may yield reward. In contrast, activity within both PFC regions is crucial for suppressing actions to obtain rewards.

\section{Active versus inhibitory learning}

When comparing the data from rats that were trained on active/ inhibitory avoidance task to those obtained from separate animals trained on the reward-seeking task, it became apparent that rats found it easier to either make an instrumental lever press or withhold it, depending on the nature of the task. To confirm these impressions statistically, we computed the number of training days rats required to achieve criterion performance ( $70 \%$ success rate) and maintain it for at least two consecutive days for each trial type for all animals included in our final analysis.

Analysis of active/inhibitory avoidance data showed that the length of training required for active and inhibitory trials differed significantly $\left(F_{(1,14)}=14.61, p<0.01\right.$; Fig. 8 , left $)$. Thus, during the initial training on active avoidance, rats required $12.1 \pm 2 \mathrm{~d}$ to achieve criterion performance. However, on introduction of the inhibitory trials in the task, rats learned to suppress lever pressing during these trials much more rapidly, requiring only $4.3 \pm 1 \mathrm{~d}$ to achieve criterion.

When an analogous analysis was performed on training data for the active/inhibitory reward-seeking task, length of training between the two trial types was again significantly different $\left(F_{(1,19)}=44.66, p<0.0001\right.$; Fig. 8 , right $)$. However, contrary to what was observed for active/inhibitory avoidance learning,

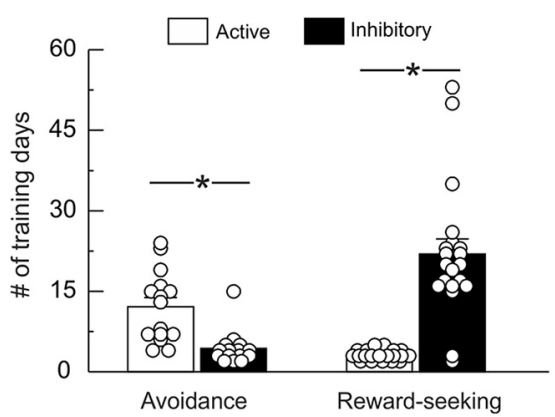

Figure 8. Number of training sessions required to obtain criterion on active versus inhibitory avoidance and reward-seeking. Number of days required to reach stable criterion performance ( $>70 \%$ success) on each trial type. During avoidance training, rats required more trials to learn an active avoidance lever-press response, compared with an inhibitory avoidance response (left). Conversely, rats required significantly more training trials to learn to withhold lever pressing to obtain reward, compared with active reward-seeking responses (right). White dots represent number of days to criterion for individual animals. Asterisk denotes $p<0.05$ between the active versus inhibitory conditions (avoidance $n=15$; reward-seeking $n=20$ ).

animals achieved criterion performance on active trials much more quickly $(3.2 \pm 0.2 \mathrm{~d})$ than when inhibitory trials were introduced $(22.0 \pm 3 \mathrm{~d})$. From these data, we conclude that rats found it more difficult to learn to lever press rather than withhold lever pressing to avoid aversive outcomes. On the other hand, when lever pressing was associated with reward delivery, inhibition of responding was more difficult and took longer to learn compared with promotion of behavior. 


\section{Discussion}

\section{Valence-dependent roles for the PL in active/inhibitory avoidance or reward-seeking}

Inactivation of the PL markedly disrupted active avoidance when animals had to discriminate between different cues informing them that either initiating or withholding a response could avoid shock. In contrast, similar treatments had no effect on active avoidance when a single cue signaled a lever press could avoid shock. These differential effects harmonize to a certain degree with somewhat conflicting findings regarding the involvement of the PL in avoidance. Thus, PL lesions did not affect acquisition of shuttle avoidance, wherein a single cue signaled rats to move between chambers to avoid shock (Moscarello and LeDoux, 2013). On the other hand, PL inactivation impaired expression of platform-mediated avoidance, when rats had to disengage from lever pressing for food to avoid shock (Bravo-Rivera et al., 2014; Diehl et al., 2018). Notably, this latter task presented rats with a motivational conflict, as avoidance required them to shift from periods of reward-seeking to a safe location away from the levers. In a similar vein, our active/inhibitory avoidance task also required shifts between producing and withholding responses. This combination of finding suggests that the PL plays a more specialized role in active avoidance, and is recruited in situations requiring flexible mitigation of conflicting motivational drives (reward-seeking vs safety) or responses (producing vs suppressing actions).

PL inactivation did not interfere with withholding of lever pressing on inhibitory avoidance trials. Superficially, this lack of effect may seem to contrast with other studies implicating this region in supressing punished responding for food or drug rewards (Resstel et al., 2008; Chen et al., 2013; Verharen et al., 2019). It is important to emphasize that the tasks used in these studies also entailed motivational conflicts, in that rewards were associated with punishment. As such, the PL may aid in suppressing punished behavior in more specialized situations involving motivational conflicts that require overriding prepotent, reward-seeking responses, whereas it does not appear necessary for inhibitory avoidance in purely aversively-motivated contexts.

The differential effects of PL inactivation on active versus inhibitory avoidance stand in striking contrast to those on active/ inhibitory reward-seeking. This experiment was designed to assess whether or not the contribution of PL to instigation and/ or inhibition of instrumental responding was specific to aversively-motivated situations. Like the avoidance task, this appetitively-motivated go/no-go task used different cues to signal a response should be produced or withheld to obtain reward. Here, PL inactivation induced an effect diametrically opposite to that observed on the avoidance task, impairing performance on inhibitory trials while having a comparatively minimal effect on active trials. This is in keeping with other reports that PL inactivation impairs response inhibition on other appetitively-motivated tasks (Ishikawa et al., 2008; Bari et al., 2011). However, when juxtaposed with the avoidance results, the contrasting effects reveal that in situations involving motivational or response conflicts, the PL aids in inhibiting actions to obtain rewards, but plays an opposite role in facilitating action to avoid negative events. More generally, these findings suggest that manner in which the PL is recruited to instigate versus suppress instrumental actions is critically dependent on the valence of the context.
Additional insight into these opposing results comes from an examination of innate response biases that emerge in aversive versus appetitive contexts. On the avoidance task, rats tended to perform better on inhibitory versus active trials, whereas on the reward-seeking task, the opposite was true, and these differences emerged early in training. This likely reflects opposing prepotent responses that are triggered in threatening and rewarding contexts. For example, aversive contexts can induce behavioral suppression, but animals learn to override this tendency as active avoidance responses emerge (Cain, 2019). On the contrary, appetitive contexts can promote approach behaviors that would need to be curtailed when learning to wait for rewards in the presence of inhibitory cues. Thus, both suppressing reward-seeking or producing active avoidance responses would require greater cognitive control to override prepotent yet incompatible behaviors. Taking this into consideration, it is reasonable to propose that the opposing effects of PL inactivation on avoidance and rewardseeking may reflect a failure of cognitive control needed to overcome competing innate drives in both aversive and appetitive situations. This notion is complementary to the proposed role for the PL in integrating contextual information to mediate response and cue conflicts (Haddon and Killcross, 2006; Marquis et al., 2007). Furthermore, it is also keeping with contemporary theory that the human anterior cingulate (homologous to the PL; Heilbronner et al., 2016) mediates cognitive control over instrumental behaviors, blunting prepotent responses when evaluating alternative choices of action (Kolling et al., 2016; Shenhav et al., 2016).

\section{IL mediation of avoidance and behavioral inhibition}

IL inactivation induced a constellation of effects distinct from PL inactivation. Foremost among these were impairments in active avoidance on both the active/inhibitory and on the single-cue avoidance tasks. This is keeping with previous studies implicating the IL in different forms of active avoidance (Martinez et al., 2013; Moscarello and LeDoux, 2013). Furthermore, they highlight a more generalized role for the IL relative to the PL in facilitating active avoidance, regardless of the presence or absence of response conflicts. It is possible that some of the effects of IL inactivation that were similar to PL inactivation may have been attributable to backflow of GABA agonist infusion from IL to $\mathrm{PL}$, which is why some groups have used angled cannulae placements when targeting the IL (Sierra-Mercado et al., 2011; BravoRivera et al., 2014; Shipman et al., 2018). However, the fact that single-cue active avoidance was impaired only by infusions of BM into IL but not PL (Moscarello and LeDoux, 2013; present study), suggests that these effects were driven primarily by suppression of activity with the more ventral $\mathrm{mPFC}$ region. In this regard, it is interesting to compare these results with an extensive literature describing a critical role for the IL in extinction of Pavlovian fear (Quirk et al., 2000; Sierra-Mercado et al., 2011; Milad and Quirk, 2012; Sangha et al., 2014). Viewed collectively, these findings indicate that whereas the IL aids in suppressing Pavlovian responses elicited by conditioned aversive stimuli during extinction, it also aids in initiating instrumental responses to avoid aversive stimuli.

In contrast to our results, Bravo-Rivera et al. (2014) reported IL inactivation did not affect signaled platform-mediated avoidance that required rats to cease reward-seeking and move to a safe location. However, these manipulations did increase freezing elicited by the warning cue, as observed by other groups (Martinez et al., 2013; Moscarello and LeDoux, 2013). These findings have led to the idea that the IL may in some instances 
enable instrumental avoidance via attenuation of Pavlovianmediated freezing to conditioned threat stimuli, which could contribute to the impairments in avoidance reported here. This being said, rats in our study received considerably more training sessions (20-40) compared with these previous studies (5-10), which presumably would have led to a greater reduction in freezing as avoidance responses became more prominent over training. In addition, although we did not explicitly measure freezing behavior, IL inactivation did not alter avoidance latencies and tended to increase locomotor activity across tasks, observations that are inconsistent with a potential increase in freezing. Alternatively, the tendency for IL inactivation to increase locomotion may have contributed to the impairments in avoidance reported here, although most of this activity likely occurred during the ITI. Furthermore, previous studies have shown that inactivation of the IL or PL does not alter activity in an open field (Fitzpatrick et al., 2011; Stevenson, 2011; Bi et al., 2013). Thus, although we cannot completely rule out alterations in freezing or locomotion may have contributed to these effects, our findings suggest that under some conditions, the IL may facilitate active avoidance in a manner relatively independent of its role in suppressing fear-related behavioral arrest.

Another key difference between the effects of PL versus IL inactivation is that the latter also impaired inhibitory avoidance. Notably, the effects of IL inactivation on both active and inhibitory avoidance were similar to those induce by inactivation of the NAc shell (Piantadosi et al., 2018), a key target of IL projections, suggesting these two regions may work in concert to facilitate opposing forms of avoidance behavior. These disinhibitory effects complement a literature implicating IL-related circuitry in suppressing different behaviors under a variety of conditions. These include the aforementioned extinction of Pavlovian fear responses, step-down passive avoidance (Jinks and McGregor, 1997), punished free-operant responding for rewards and reward consumption (Resstel et al., 2008; Jean-Richard-Dit-Bressel and McNally, 2016), extinguished instrumental drug seeking (Peters et al., 2008), and inappropriate, non-rewarded responses (Chudasama et al., 2003; Ishikawa et al., 2008; Ghazizadeh et al., 2012). With this in mind, IL inactivation also impaired inhibitory control on the reward-seeking task but, notably, did not affect lever pressing to obtain reward, although these manipulations did attenuate pressing to avoid shock. When viewing these constellations of effects within a broader framework, it would appear that the IL plays a somewhat selective role in facilitating actions to avoid noxious stimuli, but a more generalized role in inhibiting behavior in both appetitive and aversive contexts.

\section{Theoretical and clinical implications}

A primary objective of this study was to clarify how subregions of the mPFC contribute to different forms of avoidance. However, these findings provide novel insight into the complex manner in which the PL and IL either initiate or suppress goaldirected actions. Based in part on studies of Pavlovian fear conditioning and drug self-administration, a conceptual framework emerged positing the PL may be part of a "go" circuit that initiates goal-directed action, whereas IL-related circuits may subserve a "stop" function, regulating behavioral suppression (Peters et al., 2008; Sotres-Bayon and Quirk, 2010; Gass and Chandler, 2013). Recently, however, this elementary dichotomy of function has been scrutinized (Moorman and Aston-Jones, 2015; Moorman et al., 2015). Indeed, during free-operant responding for natural rewards, PL inactivation actually increase reward-seeking, whereas both PL and IL inactivation decreased seeking during extinction (Caballero et al., 2019). Our results call for additional refinement of this conceptualization. For example, our observations indicate that the PL facilitates flexible instigation or inhibition of instrumental action, in a manner critically dependent on whether they may avoid aversive outcomes or procure rewards. This in turn may relate to the level of cognitive control required to overcome innate prepotent responses. Furthermore, our findings bolster a considerable literature identifying a key role for the IL in maximizing behavioral utility by suppressing Pavlovian or instrumental behavior in both appetitive and aversive context. Yet they further highlight that this region may be recruited to initiate actions in the service of avoiding aversive stimuli. The possibility remains that facilitation versus inhibition of action by the PL or IL under different conditions may be mediated by distinct ensembles of PFC projection neurons with different downstream targets (Warren et al., 2019).

Studies in healthy humans have implicated the mPFC in regulating avoidance responses (Mobbs et al., 2007; Aupperle et al., 2015; Chrysikou et al., 2017). Conversely, maladaptive exaggerated defensive reactions and avoidance behaviors are characteristic features of numerous neuropsychiatric conditions, including PTSD (Pineles et al., 2011), depression (Ottenbreit et al., 2014), and anxiety disorders (Heuer et al., 2007). These conditions are often associated with abnormal profiles of prefrontal activation in response to aversive stimuli (White et al., 2017; Kunimatsu et al., 2019; Ironside et al., 2020). The preclinical findings reported her provide novel insight into how distinct regions of the $\mathrm{mPFC}$ may regulate different aspects of avoidance behavior and in turn, may aid in elucidating how prefrontal pathophysiology may contribute to these abnormal profiles of avoidance associated with these illnesses.

\section{References}

Aupperle RL, Melrose AJ, Francisco A, Paulus MP, Stein MB (2015) Neural substrates of approach-avoidance conflict decision-making. Hum Brain Mapp 36:449-462.

Bari A, Robbins TW (2013) Inhibition and impulsivity: behavioral and neural basis of response control. Prog Neurobiol 108:44-79.

Bari A, Mar AC, Theobald DE, Elands SA, Oganya K, Eagle DM, Robbins TW (2011) Prefrontal and monoaminergic contributions to stop-signal task performance in rats. J Neurosci 31:9254-9263.

Beck KD, Jiao X, Ricart TM, Myers CE, Minor TR, Pang KC, Servatius RJ (2011) Vulnerability factors in anxiety: strain and sex differences in the use of signals associated with non-threat during the acquisition and extinction of active-avoidance behavior. Prog Neuropsychopharmacol Biol Psychiatry 35:1659-1670.

Berger DF, Brush FR (1975) Rapid acquisition of discrete-trial lever-press avoidance: effects of signal-shock interval. J Exp Anal Behav 24:227-239.

Bi LL, Wang J, Luo ZY, Chen SP, Geng F, Chen YH, Li SJ, Yuan CH, Lin S, Gao TM (2013) Enhanced excitability in the infralimbic cortex produces anxiety-like behaviors. Neuropharmacology 72:148-156.

Bravo-Rivera C, Roman-Ortiz C, Brignoni-Perez E, Sotres-Bayon F, Quirk GJ (2014) Neural structures mediating expression and extinction of platform-mediated avoidance. J Neurosci 34:9736-9742.

Brennan JF, Wisniewski C (1982) The efficacy of response prevention on avoidance behavior in young and adult rats with prefrontal cortical injury. Behav Brain Res 4:117-131.

Brito GN, Brito LS (1990) Septohippocampal system and the prelimbic sector of frontal cortex: a neuropsychological battery analysis in the rat. Behav Brain Res 36:127-146.

Brog JS, Salyapongse A, Deutch AY, Zahm DS (1993) The patterns of afferent innervation of the core and shell in the accumbens part of the rat ventral striatum: immunohistochemical detection of retrogradely transported fluoro-gold. J Comp Neurol 338:255-278.

Burgos-Robles A, Kimchi EY, Izadmehr EM, Porzenheim MJ, Ramos-Guasp WA, Nieh EH, Felix-Ortiz AC, Namburi P, Leppla CA, Presbrey KN, Anandalingam KK, Pagan-Rivera PA, Anahtar M, Beyeler A, Tye KM (2017) Amygdala inputs to prefrontal cortex guide behavior amid conflicting cues of reward and punishment. Nat Neurosci 20:824-835. 
Caballero JP, Scarpa GB, Remage-Healey L, Moorman DE (2019) Differential effects of dorsal and ventral medial prefrontal cortex inactivation during natural reward-seeking, extinction, and cue-induced reinstatement. eNeuro 6:ENEURO.0296-19.2019.

Cain CK (2019) Avoidance problems reconsidered. Curr Opin Behav Sci 26:9-17.

Chen BT, Yau H-J, Hatch C, Kusumoto-Yoshida I, Cho SL, Hopf FW, Bonci A (2013) Rescuing cocaine-induced prefrontal cortex hypoactivity prevents compulsive cocaine seeking. Nature 496:359-362.

Chrysikou EG, Gorey C, Aupperle RL (2017) Anodal transcranial direct current stimulation over right dorsolateral prefrontal cortex alters decision making during approach-avoidance conflict. Soc Cogn Affect Neurosci 12:468-475.

Chudasama Y, Robbins TW (2003) Dissociable contributions of the orbitofrontal and infralimbic cortex to Pavlovian autoshaping and discrimination reversal learning: further evidence for the functional heterogeneity of the rodent frontal cortex. J Neurosci 23:8771-8780.

Chudasama Y, Passetti F, Rhodes SE, Lopian D, Desai A, Robbins T (2003) Dissociable aspects of performance on the 5-choice serial reaction time task following lesions of the dorsal anterior cingulate, infralimbic and orbitofrontal cortex in the rat: differential effects on selectivity, impulsivity and compulsivity. Behav Brain Res 146:105-119.

Dalton GL, Wang NY, Phillips AG, Floresco SB (2016) Multifaceted contributions by different regions of the orbitofrontal and medial prefrontal cortex to probabilistic reversal learning. J Neurosci 36:1996-2006.

Diehl MM, Bravo-Rivera C, Rodriguez-Romaguera J, Pagan-Rivera PA, Burgos-Robles A, Roman-Ortiz C, Quirk GJ (2018) Active avoidance requires inhibitory signaling in the rodent prelimbic prefrontal cortex. Elife 7:e34657.

Dinsmoor JA (2001) Stimuli inevitably generated by behavior that avoids electric shock are inherently reinforcing. J Exp Anal Behav 75:311-333.

Fernando ABP, Urcelay GP, Mar AC, Dickinson A, Robbins TW (2014) Safety signals as instrumental reinforcers during free-operant avoidance. Learn Mem 21:488-497.

Fernando ABP, Mar AC, Urcelay GP, Dickinson A, Robbins TW (2015) Avoidance behavior: a free-operant lever-press avoidance task for the assessment of the effects of safety signals. Curr Protoc Neurosci 70:8-32.

Fitzpatrick CJ, Knox D, Liberzon I (2011) Inactivation of the prelimbic cortex enhances freezing induced by trimethylthiazoline, a component of fox feces. Behav Brain Res 221:320-323.

Floresco SB, Montes DR, Tse MMT, van Holstein M (2018) Differential contributions of nucleus accumbens subregions to cue-guided risk/reward decision making and implementation of conditional rules. J Neurosci 38:1901-1914.

Gass JT, Chandler LJ (2013) The plasticity of extinction: contribution of the prefrontal cortex in treating addiction through inhibitory learning. Front Psychiatry 4:46.

Ghazizadeh A, Ambroggi F, Odean N, Fields HL (2012) Prefrontal cortex mediates extinction of responding by two distinct neural mechanisms in accumbens shell. J Neurosci 32:726-737.

Groenewegen HJ, Wright CI, Beijer AVJ, Voorn P (1999) Convergence and segregation of ventral striatal inputs and outputs. Ann NY Acad Sci 877:49-63.

Haddon JE, Killcross S (2006) Prefrontal cortex lesions disrupt the contextual control of response conflict. J Neurosci 26:2933-2940.

Heilbronner SR, Rodriguez-Romaguera J, Quirk GJ, Groenewegen HJ, Haber SN (2016) Circuit-based corticostriatal homologies between rat and primate. Biol Psychiatry 80:509-521.

Heinsbroek RP1, Feenstra MG, Boon P, Van Haaren F, Van de Poll NE (1988) Sex differences in passive avoidance depend on the integrity of the central serotonergic system. Pharmacol Biochem Behav 31:499-503.

Heuer K, Rinck M, Becker ES (2007) Avoidance of emotional facial expressions in social anxiety: the approach-avoidance task. Behav Res Ther 45:2990-3001

Holson RR (1986) Mesial prefrontal cortical lesions and timidity in rats. I. Reactivity to aversive stimuli. Physiol Behav 37:221-230.

Hurley KM, Herbert H, Moga MM, Saper CB (1991) Efferent projections of the infralimbic cortex of the rat. J Comp Neurol 308:249-276.

Ironside M, Amemori KI, McGrath CL, Pedersen ML, Kang MS, Amemori S, Frank MJ, Graybiel AM, Pizzagalli DA (2020) Approach-avoidance conflict in major depressive disorder: congruent neural findings in humans and nonhuman Primates. Biol Psychiatry 87:399-408.
Ishikawa A, Ambroggi F, Nicola SM, Fields HL (2008) Contributions of the amygdala and medial prefrontal cortex to incentive cue responding. Neuroscience 155:573-584.

Jean-Richard-Dit-Bressel P, McNally GP (2016) Lateral, not medial, prefrontal cortex contributes to punishment and aversive instrumental learning. Learn Mem 23:607-617.

Jinks AL, McGregor IS (1997) Modulation of anxiety-related behaviours following lesions of the prelimbic or infralimbic cortex in the rat. Brain Res 772:181-190.

Joel D, Tarrasch R, Feldon J, Weiner I (1997) Effects of electrolytic lesions of the medial prefrontal cortex or its subfields on 4 -arm baited, 8 -arm radial maze, two-way active avoidance and conditioned fear tasks in the rat. Brain Res 765:37-50.

Kolling N, Behrens TEJ, Wittmann MK, Rushworth MFS (2016) Multiple signals in anterior cingulate cortex. Curr Opin Neurobiol 37:36-43.

Kunimatsu A, Yasaka K, Akai H, Kunimatsu N, Abe O (2019) MRI findings in posttraumatic stress disorder. J Magn Reson Imaging. Advance online publication. Retrieved September 12, 2019. doi: 10.1002/jmri.26929.

LeDoux JE, Moscarello J, Sears R, Campese V (2017) The birth, death and resurrection of avoidance: a reconceptualization of a troubled paradigm. Mol Psychiatry 22:24-36.

Levita L, Hoskin R, Champi S (2012) Avoidance of harm and anxiety: a role for the nucleus accumbens. Neuroimage 62:189-198.

Marquis JP, Killcross S, Haddon JE (2007) Inactivation of the prelimbic, but not infralimbic, prefrontal cortex impairs the contextual control of response conflict in rats. Eur J Neurosci 25:559-566.

Martinez RCR, Gupta N, Lázaro-Muñoz G, Sears RM, Kim S, Moscarello JM, LeDoux JE, Cain CK (2013) Active vs. reactive threat responding is associated with differential c-Fos expression in specific regions of amygdala and prefrontal cortex. Learn Mem 20:446-452.

Martin JH, Ghez C (1999) Pharmacological inactivation in the analysis of the central control of movement. J Neurosci Methods 86:145-159.

Milad MR, Quirk GJ (2012) Fear extinction as a model for translational neuroscience: ten years of progress. Annu Rev Psychol 63:129-151.

Mobbs D, Petrovic P, Marchant JL, Hassabis D, Weiskopf N, Seymour B, Dolan RJ, Frith CD (2007) When fear is near: threat imminence elicits prefrontal-periaqueductal gray shifts in humans. Science 317:1079-1083.

Moorman DE, Aston-Jones G (2015) Prefrontal neurons encode contextbased response execution and inhibition in reward-seeking and extinction. Proc Natl Acad Sci USA 112:9472-9477.

Moorman DE, James MH, McGlinchey EM, Aston-Jones G (2015) Differential roles of medial prefrontal subregions in the regulation of drug seeking. Brain Res 1628:130-146.

Moscarello JM, LeDoux JE (2013) Active avoidance learning requires prefrontal suppression of amygdala-mediated defensive reactions. J Neurosci 33:3815-3823.

Ottenbreit ND, Dobson KS, Quigley L (2014) An examination of avoidance in major depression in comparison to social anxiety disorder. Behav Res Ther 56:82-90.

Peters J, LaLumiere RT, Kalivas PW (2008) Infralimbic prefrontal cortex is responsible for inhibiting cocaine seeking in extinguished rats. J Neurosci 28:6046-6053.

Piantadosi PT, Yeates DCM, Floresco SB (2018) Cooperative and dissociable involvement of the nucleus accumbens core and shell in the promotion and inhibition of actions during active and inhibitory avoidance. Neuropharmacology 138:57-71.

Pineles SL, Mostoufi SM, Ready CB, Street AE, Griffin MG, Resick PA (2011) Trauma reactivity, avoidant coping, and PTSD symptoms: a moderating relationship? J Abnorm Psychol 120:240-246.

Quirk GJ, Russo GK, Barron JL, Lebron K (2000) The role of ventromedial prefrontal cortex in the recovery of extinguished fear. J Neurosci 20:6225-6231.

Ramirez F, Moscarello JM, LeDoux JE, Sears RM (2015) Active avoidance requires a serial basal amygdala to nucleus accumbens shell circuit. J Neurosci 35:3470-3477.

Resstel LBM, Souza RF, Guimarães FS (2008) Anxiolytic-like effects induced by medial prefrontal cortex inhibition in rats submitted to the Vogel conflict test. Physiol Behav 93:200-205.

Sangha S, Robinson PD, Greba Q, Davies DA, Howland JG (2014) Alterations in reward, fear and safety cue discrimination after inactivation of the rat prelimbic and infralimbic cortices. Neuropsychopharmacology 39:2405-2413. 
Schlund MW, Magee S, Hudgins CD (2011) Human avoidance and approach learning: evidence for overlapping neural systems and experiential avoidance modulation of avoidance neurocircuitry. Behav Brain Res 225:437448.

Schlund MW, Brewer AT, Magee SK, Richman DM, Solomon S, Ludlum M, Dymond S (2016) The tipping point: value differences and parallel dorsal-ventral frontal circuits gating human approach-avoidance behavior. Neuroimage 136:94-105.

Schwartz N, Miller C, Fields HL (2017) Cortico-accumbens regulation of approach-avoidance behavior is modified by experience and chronic pain. Cell Rep 19:1522-1531.

Shenhav A, Cohen JD, Botvinick MM (2016) Dorsal anterior cingulate cortex and the value of control. Nat Neurosci 19:1286-1291.

Shipman ML, Trask S, Bouton ME, Green JT (2018) Inactivation of prelimbic and infralimbic cortex respectively affects minimally-trained and extensively-trained goal-directed actions. Neurobiol Learn Mem 155:164-172.

Sierra-Mercado D, Padilla-Coreano N, Quirk GJ (2011) Dissociable roles of prelimbic and infralimbic cortices, ventral hippocampus, and basolateral amygdala in the expression and extinction of conditioned fear. Neuropsychopharmacology 36:529-538.

Sotres-Bayon F, Quirk GJ (2010) Prefrontal control of fear: more than just extinction. Curr Opin Neurobiol 20:231-235.
Stevenson CW (2011) Role of amygdala-prefrontal cortex circuitry in regulating the expression of contextual fear memory. Neurobiol Learn Mem 96:315-323.

St. Onge JR, Floresco SB (2010) Prefrontal cortical contribution to risk-based decision making. Cereb Cortex 20:1816-1828.

van Holstein M, Floresco SB (2020) Dissociable roles for the ventral and dorsal medial prefrontal cortex in cue-guided risk/reward decision making. Neuropsychopharmacology 45:683-693.

Verharen JPH, van den Heuvel MW, Luijendijk M, Vanderschuren L, Adan RAH (2019) Corticolimbic mechanisms of behavioral inhibition under threat of punishment. J Neurosci 39:4353-4364.

Vertes RP (2004) Differential projections of the infralimbic and prelimbic cortex in the rat. Synapse 51:32-58.

Warren BL, Kane L, Venniro M, Selvam P, Quintana-Feliciano R, Mendoza MP, Madangopal R, Komer L, Whitaker LR, Rubio FJ, Bossert JM, Caprioli D, Shaham Y, Hope BT (2019) Separate vmPFC ensembles control cocaine self-administration versus extinction in rats. J Neurosci 39:7394-7407.

White SF, Geraci M, Lewis E, Leshin J, Teng C, Averbeck B, Meffert H, Ernst M, Blair JR, Grillon C, Blair KS (2017) Prediction error representation in individuals with generalized anxiety disorder during passive avoidance. Am J Psychiatry 174:110-117 